\title{
Impact of temperature and concentration dispersion on the physiology of blood nanofluid: links to atherosclerosis
}

\author{
$\mathrm{K}^{\mathrm{M}}$ SURABHI ${ }^{1}$, J V RAMANA REDDY ${ }^{2}$ and D SRIKANTH ${ }^{1, *}$ \\ ${ }^{1}$ Department of Applied Mathematics, Defence Institute of Advanced Technology (Deemed University), \\ Pune 411025, India \\ ${ }^{2}$ Department of Mathematics, Indian Institute of Technology Madras, Chennai 600036, India \\ e-mail: sri_dasari1977@yahoo.co.in
}

MS received 19 February 2018; revised 23 May 2018; accepted 26 June 2018; published online 30 November 2018

\begin{abstract}
A theoretical model of blood-silver nanofluid flow through an $\omega$-shaped tapered stenotic artery in the presence of a catheter is understood in detail. The rheology of the blood is considered as that of a micro-polar fluid. Suspension of the silver nano-particles in the micro-polar fluid is proposed to investigate the temperature and concentration dispersion from the immersed temperature-sensitive drug-coated nano-particles. The effects of velocity discontinuity at the arterial wall in the stenotic and non-stenotic regions are considered. The outer surface of the catheter is layered with the temperature-sensitive, drug-coated nano-particles. The resulting mathematical formulation involving the coupled non-linear momentum, temperature and concentration equations is solved using the Homotopy Perturbation Method. The efficiency and convergence of the method to the modelled equations are discussed in detail. The consequent effects of the fluid and geometric parameters on pressure drop, flow rate, impedance and wall shear stress of the fluid flow are computed. It is noticed that the high volume fraction of the nano-particles in the blood results in high flow velocity, contributing to secondary flow regions, thus resulting in higher shear stress. Such high volume fraction of the nano-particle may lead to the pathological disorder called aneurysm. This physical model has an important application of drug delivery in biomedical and pharmaceutical industry to prevent obstructions in arteries. Further, the results obtained could be very useful in the manufacturing of related artificial devices.
\end{abstract}

Keywords. Micro-polar nanofluid; stenotic artery; homotopy perturbation method; impedance; wall shear stress.

\section{Introduction}

Physiological studies based on the blood flow through arteries and veins in the complex network of the living life are the prime concern of scientist in the Bio-mechanics area. According to the World Health Organization (WHO), in $2012,68 \%$ of the deaths are due to the non-communicable diseases (NCDs), out of which one-third are due to cardiovascular diseases (CVDs) [1]. Disorders of the heart and blood vessel that come under CVDs are bifurcated as coronary heart disease (CHD), cerebrovascular disease, rheumatic heart disease, etc. Based on reports of epidemiological studies [2] and those of NCD based on the Global Burden of Diseases (GBD) by [3], it is clear that there is a continuous increase of NCDs in India, the main contributor of which is CVDs. According to Fung [4], human circulation system is a vast network of organs and vessels that are responsible for the flow of blood, nutrients and oxygen, and excretion of metabolic waste from the living cells.

*For correspondence
Reduction of blood flow in coronary artery leads to myocardial infarction. CHD occurs due to vascular dysfunction, which is accounted for as atherosclerosis, thrombosis or ischemia. Atherosclerosis means narrowing of the artery lumen, medically termed as stenosis, which happens due to the deposition of cholesterol, calcium, abnormal growth of tissues, fatty substances and fibrin (clot in the blood) in the inner lining of the artery. Due to the formation of the stenosis, there is a significant alteration in the blood flow pattern and fluid dynamic properties.

Blood, a marvelous fluid, which nurtures life, is an aqueous solution (plasma) with suspension of a variety of cells. A good number of studies considered Newtonian behaviour of the blood. Young and Tsai [5] and Bluestein et al [6] investigated the hydrodynamic characteristics in a healthy blood vessel, in which they considered the laminar and turbulent behaviour, for steady case with respect to the Reynolds number ranging from 100 to 5000 and 300 to 3600, respectively. Ariman et al [7] reviewed the macrocontinuum approach of the fluid flow and concluded that the deformation in blood vessel results in a change in the 
mechanical properties, which occurs due to the rotational velocities of rigid neutrally buoyant spheres under some flow conditions. Schmid-Schönbein et al [8] understood that the interaction between the blood components like leukocytes and erythrocytes in the capillary is the primary cause of non-Newtonian behaviour of blood flow. Reddy et al [9] modelled the blood as a couple stress fluid and concluded that it is a couple stress fluid having high impedance as compared with the classical Newtonian fluid. Some authors $[10,11]$ theoretically studied the blood rheology on different kinds of obstructions like cerebral aneurysm and coronary artery disease (e.g., stenotic), respectively.

Among the several non-Newtonian fluid models, the model developed by Eringen [12] in 1964 exhibits microrotational effects, due to the presence of couple stress and body couples. Micro-polar fluids accommodate similar rheological properties of the fluid like, animal blood, polymeric fluids and liquid crystals. Rheological properties of blood resemble those of micro-polar fluids. Several investigators $[13,14]$ considered micro-polar fluids in their modelling for blood flow analysis.

The procedures like angioplasty, ventri-culogram and catheterization are developed to treat stenosed blood vessels. Among these methods, the catheterization is the cheapest procedure, which is used to remove the formation of clogging in arteries by inserting a long, thin, flexible tube (called a catheter) into the lumen of the unhealthy blood vessel. The insertion of the catheter into the blood vessel results in changes in blood flow dynamics in the entire region. Therefore investigating the catheter influence on the rheological properties of the blood flow is vital. Roos and Lykoudis [15] examined the pressure distribution in the presence of the catheter by considering the peristaltic motion through the infinitely long cylinders. McDonald [16] studied the peristaltic flow of blood and explored the alteration in pressure gradient when a catheter is inserted into a femoral artery. Dash et al [17] studied the blood flow dynamics in a stenotic curved artery along with the effects of a catheter. Srinivasacharya and Srikanth [18] studied the oscillatory flow of micro-polar fluid through a mild contracted annulus in the stenotic artery and calculated wall shear stress and impedance. Reddy and Srikanth [19] studied a mathematical model of micro-polar fluid through overlapping tapered stenotic artery in the presence of the catheter with slip velocity. Mekheimer and co-workers contributed deeply towards blood flow through arteries through the articles [20-22] and [23].

Recently, pharmaceutical research exposed local drug delivery procedure to the targeted organs. The procedure has been used to prevent the growth and formation of the stenosis in the lumen of the arterial surface. Currently, clinical use of drug-eluting stents (DESs) is not found to be efficacious in the peripheral circulation. Further pursuits led to the development of drug-eluting balloons (DEBs) technology, which overcame the limitations of DESs. DEBs elute an anti-proliferative agent and are designed to compress and deform the plaque. In advanced therapy and diagnostics, nano-medicine is an emerging field that utilizes nanotechnology. Based on the literature survey, these engineered colloids have numerous applications such as those in Micro-Electro-Mechanical Systems (MEMS), Nano-Electro-Mechanical Systems (NEMS), biomedical engineering (imaging and ablation), nuclear reactors, extraction of geothermal power, etc. [24, 25]. Nanofluid is a mixture of nano-sized particles suspended in the base fluid, which possesses changes in thermo-physical properties such as thermal conductivity, thermal diffusivity, viscosity and convective heat transfer coefficients. These nano-particles have unique features and are stable in base fluids. Nano-coated drug particles increase the thermal conductivity with the increase of volume concentration. This expected enhancement is diminished at a higher volume concentration, but viscosity increases. For prevention of CVDs, detection of the dysfunctional endothelium can be possible with the nano-particle drug targeted to the stenotic region of the artery. The mathematical and computational model determines the diffusion and convection process.

Analytical techniques for solving the non-linear problems is still dominating the area of researchers. Perturbation techniques are used widely, but they having their own limitations. Small-parameter assumption for the perturbation technique is very crucial. To avoid this assumption, Liao [26] proposed a non-linear asymptotic technique, which is based on homotopy, and does not require small and large parameters. The homotopy technique transforms the non-linear problems into an infinite number of linear problems. Perturbation methods by Nayfeh [27], homotopy analysis methods by Shijun [28] and variational iteration methods by He [29] also have some limitations. Later on, He [30] proposed a new method based on properties of homotopy and perturbation methods, named as Homotopy Perturbation Method (HPM), with an embedding parameter. The solutions obtained by HPM are uniformly valid for parameters, irrespective of the nature of the parameter. Elnaqeeb et al [31] studied the impact of Copper nanoparticle volume fraction on the blood flow dynamics, during its flow through an asymmetric stenotic and catheterized arterial segment. Ellahi [32] studied the mathematical model for viscous fluid with nano-particles embedded in it.

This paper presents an analytical study of blood flow through a stenotic tapered artery with the inclusion of a druglayered catheter. In this model, we considered the heterogeneous velocity slip at the lumen of the arterial surface. Fluid flow is modelled with a micro-polar fluid and metallic silver nano-particles immersed in it. Non-linear coupled equations are solved using the HPM. The effects of thermophoresis, Brownian motion, volume fraction of the nanoparticles and other parameters arising out of the model are considered on temperature, concentration, axial velocity and 
micro-rotational profiles. Also studied are pressure drop, Wall shear stress and impedance on the fluid flow.

\section{Mathematical modelling and the governing equations}

\subsection{Geometry description}

The steady, laminar and incompressible silver nano-particles immersed in a micro-polar fluid flowing through an $\omega$ shaped stenotic tapered artery in the presence of a catheter is discussed. Schematic diagram of the same is as shown in figure 1. Let $(r, \theta, z)$ be the cylindrical polar coordinates, where $z$ is taken along the axis of the artery; $r$ and $\theta$ quantify the radial and circumferential directions, respectively. The fluid flows through the annular region formed due to the catheter of radius $\left(r_{c}\right)$ and varying annular radius $(h(z))$. Here, $z_{C}$ indicates the location on $z$-axis corresponding to the critical height of the overlapping stenosis; $z_{L}$ and $z_{R}$ correspond to the locations where maximum heights of the stenosis occur. The length of the arterial segment in the pre-stenotic region is represented as $L_{0}, L_{1}$ depicts the length of the stenosis and the equation of varying annular radius is as given by Reddy and Srikanth [19]:

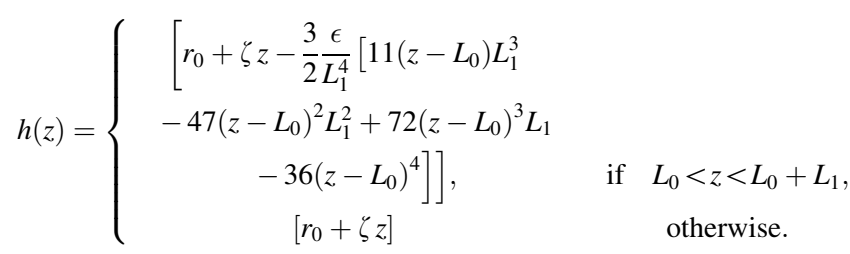

Furthermore, $\zeta(=\tan (\psi))$ is the tapering parameter and $\psi$ is the corresponding tapering angle; $\psi(>0$ and $<0)$ correspond, respectively, to the diverging and converging nature of the artery, whereas $\psi(=0)$ indicates the nontapered nature of the artery; $\epsilon$ is the maximum height of the

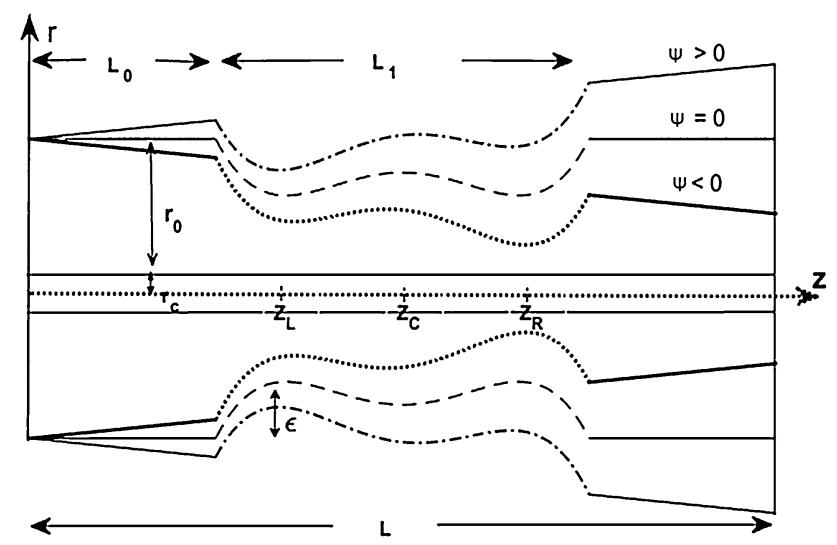

Figure 1. Geometry of the overlapping stenotic tapered artery with catheter. stenosis, which protrudes into the lumen of the annular region. The radius of the annular region in case of nontapered $(\zeta=0)$ and non-stenotic $(\epsilon=0)$ region is indicated as $r_{0}$, which can be obtained by Eq. (1).

\subsection{Physical properties of nanofluid}

Blood is a suspension of cells or particles in a complex, continuous, aqueous solution, called plasma. The cells consist of a variety of blood cells, e.g., red blood cells (erythrocytes), white blood cells (leukocytes) and platelets. The suspending medium plasma is composed of water (90\%), plasma protein $(7 \%)$ and other inorganic and organic substances (3\%). Red blood cells occupy about $97 \%$ of the total cell volume in blood; their shape, size, deformation and flexibility play a vital role in the rheology of blood. Haematocrit measures the volume percentage of red blood cells in plasma [4]. Density- and temperaturedependent physical properties like specific heat capacity and thermal expansion coefficients of the micro-polar fluid based on nano-particle volume fraction relations are as follows:

$$
\begin{gathered}
\rho_{n f}=\rho_{f}(1-\phi)+\rho_{p} \phi, \\
\left(\rho c_{p}\right)_{n f}=\left(\rho c_{p}\right)_{f}(1-\phi)+\left(\rho c_{p}\right)_{p} \phi \\
(\rho \gamma)_{n f}=(\rho \gamma)_{f}(1-\phi)+(\rho \gamma)_{p} \phi .
\end{gathered}
$$

The nano-particles are uniformly dispersed and are stable in the base fluid. The relative change in the concentration of the nano-particles with respect to the base fluid in parabolic nature is studied by many researchers. Taking into account the volume fraction of the nano-particles in the base fluid, the viscosity of the nanofluids has been suggested by many researchers. Firstly, Einstein [33], in his theory of Brownian motion, studied the effect on the motion of a liquid with very small spheres suspended in it. He gave the following relation, which is valid for low volume fraction $(\phi<0.02)$ of the suspended spheres:

$$
\mu_{n f}=\mu_{f}(1+2.5 \phi) .
$$

The classical Brinkman model [34] studied the viscosity of concentrated suspensions and solutions using the relation

$$
\mu_{n f}=\mu_{f} /(1-\phi)^{2.5}
$$

Subsequently, Batchelor [35] also considered the bulk stress, which appeared because of Brownian motion in the dilute case, where the effective viscosity is considered as

$$
\mu_{n f}=\mu_{f}\left(1+2.5 \phi+6.2 \phi^{2}\right) .
$$

All these models are considered as classical models and extensively used for the numerical simulations. However, 
they are not appropriate for all types of nanofluids. For different nano-particles, empirical viscosity is demonstrated by researchers through experiments. The present model investigates the variations in the flow characteristics due to the suspension of various nano-particles, which are Copper nano-particles, Aluminium Oxide nano-particles, Titanium Oxide nano-particles and silver nano-particles immersed in blood once at a time, and the corresponding relations are presented in table 1 . In addition, the dependence of vortex viscosity on the nanoparticle volume fraction is also formulated and presented in the table.

\subsection{Governing equations}

Consider the mathematical model of an axisymmetric flow of a steady, incompressible, metallic nano-particles blood flow in an annular region $\left[r_{c}, h(z)\right]$. It is assumed that there is no chemical reaction between the fluid and nano-particles; further, it is presumed that they are in thermal equilibrium. The governing equations for the steady, nanoparticle-immersed incompressible micro-polar fluid flow in the presence of body forces and absence of body couple are

$$
\nabla \cdot \bar{V}=0
$$

$$
\rho_{n f} \frac{D \bar{V}}{D t}=-\nabla P+2 \eta_{n f}(\nabla \times \bar{G})+\left(\mu_{n f}+\eta_{n f}\right) \nabla^{2} \bar{V}+F
$$

$$
\begin{gathered}
\rho_{n f} J \frac{D \bar{G}}{D t}=2 \eta_{n f}(\nabla \times \bar{V})-4 \eta_{n f} \bar{G}+4 \Gamma \nabla^{2} \bar{G} \\
\frac{D T}{D t}=\frac{\kappa_{n f}}{\left(\rho c_{p}\right)_{n f}} \nabla^{2} T+\frac{\left(\rho c_{f}\right)_{n f}}{\left(\rho c_{p}\right)_{n f}} \\
{\left[D_{B}(\nabla C \cdot \nabla T)+\frac{D_{T}}{T_{1}}(\nabla T \cdot \nabla T)\right]} \\
\frac{D C}{D t}=D_{B} \nabla^{2} C+\frac{D_{T}}{T_{1}} \nabla^{2} T
\end{gathered}
$$

where $\bar{V}=(u, 0, v)$ is the velocity vector and the angular momentum is $\bar{G}=(0, \omega, 0) ; u, v$ and $\omega$ are functions of $r$ and $z$. Micro-inertia is denoted by $J$ and $\Gamma$ is the material constant. The fluid density of the nanofluid and fluid pressure are represented by $\rho_{n f}$ and $P$, respectively. The buoyancy force is represented as $F$; $\mu_{n f}$ represents the dynamic viscosity and $\eta_{n f}$ indicates the vortex viscosity of the considered nanofluid. $T$ and $C$ are the temperature and concentration, respectively, of the nanofluid. $D_{B}$ is Brownian diffusion coefficient and $D_{T}$ is thermophoretic diffusion coefficient.

The governing equations (7)-(11) in component form are as follows:

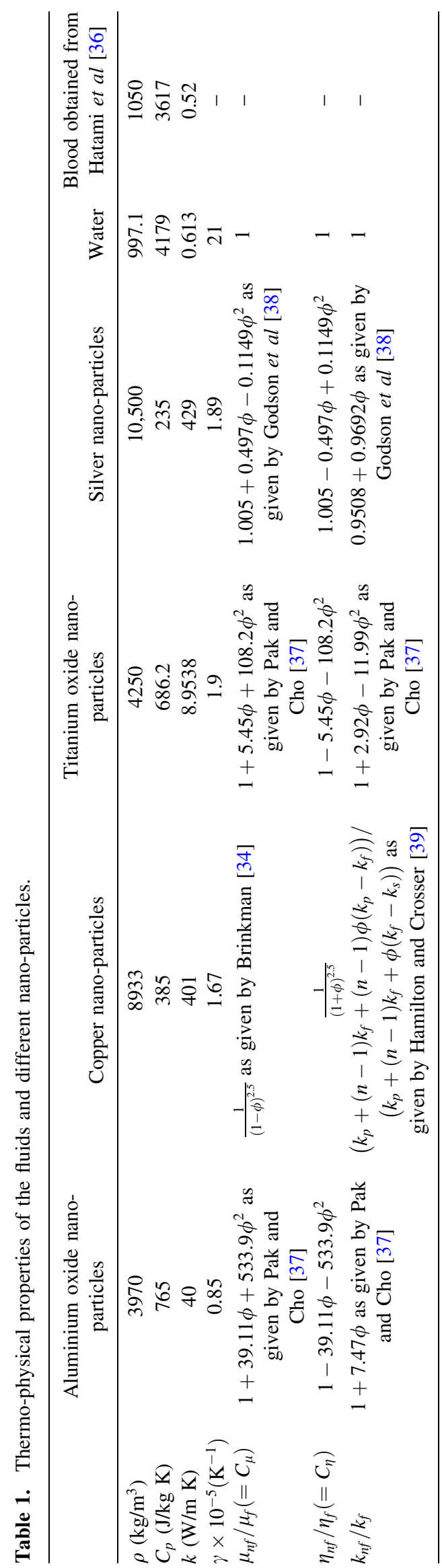




$$
\begin{aligned}
& \frac{\partial u}{\partial r}+\frac{u}{r}+\frac{\partial v}{\partial z}=0 \\
& \rho_{n f}\left(u \frac{\partial u}{\partial r}+v \frac{\partial u}{\partial z}\right)=-\frac{\partial P}{\partial r}-2 \eta_{n f}\left(\frac{\partial \omega}{\partial z}\right) \\
& +\left(\mu_{n f}+\eta_{n f}\right)\left(\frac{\partial^{2} u}{\partial r^{2}}+\frac{\partial^{2} u}{\partial z^{2}}-\frac{u}{r^{2}}+\frac{1}{r} \frac{\partial u}{\partial r}\right), \\
& \rho_{n f}\left(u \frac{\partial v}{\partial r}+v \frac{\partial v}{\partial z}\right)=-\frac{\partial P}{\partial z}+2 \eta_{n f}\left(\frac{\partial \omega}{\partial r}+\frac{\omega}{r}\right)+\left(\mu_{n f}+\eta_{n f}\right) \\
& \left(\frac{\partial^{2} v}{\partial r^{2}}+\frac{1}{r} \frac{\partial v}{\partial r}+\frac{\partial^{2} v}{\partial z^{2}}\right)+g(\rho \gamma)_{n f}\left(T-T_{1}\right)+g(\rho \gamma)_{n f}\left(C-C_{1}\right), \\
& \rho_{n f} J\left(u \frac{\partial \omega}{\partial r}+v \frac{\partial \omega}{\partial z}\right)=2 \eta_{n f}\left(\frac{\partial v}{\partial z}-\frac{\partial v}{\partial r}\right) \\
& -4 \eta_{n f} \omega+4 \Gamma\left(\frac{\partial^{2} \omega}{\partial r^{2}}+\frac{\partial^{2} \omega}{\partial z^{2}}+\frac{1}{r} \frac{\partial \omega}{\partial r}-\frac{\omega}{r^{2}}\right), \\
& \left(u \frac{\partial T}{\partial r}+v \frac{\partial T}{\partial z}\right)=\alpha_{n f}\left(\frac{\partial^{2} T}{\partial r^{2}}+\frac{1}{r} \frac{\partial T}{\partial r}+\frac{\partial^{2} T}{\partial z^{2}}\right) \\
& +\tau_{n f}\left(D_{B}\left(\frac{\partial C}{\partial r} \frac{\partial T}{\partial r}+\frac{\partial C}{\partial z} \frac{\partial T}{\partial z}\right)+\frac{D_{T}}{T_{1}}\left(\left(\frac{\partial T}{\partial r}\right)^{2}+\left(\frac{\partial T}{\partial z}\right)^{2}\right)\right), \\
& \left(u \frac{\partial C}{\partial r}+v \frac{\partial C}{\partial z}\right)=D_{B}\left(\frac{\partial^{2} C}{\partial r^{2}}+\frac{1}{r} \frac{\partial C}{\partial r}+\frac{\partial^{2} C}{\partial z^{2}}\right) \\
& +\frac{D_{T}}{T_{1}}\left(\frac{\partial^{2} T}{\partial r^{2}}+\frac{1}{r} \frac{\partial T}{\partial r}+\frac{\partial^{2} T}{\partial z^{2}}\right) .
\end{aligned}
$$

$T_{1}$ and $C_{1}$ are the temperature and concentration at the artery wall, respectively. Thermal conductivity of nanofluid is $\kappa_{n f}$, specific heat capacitance of the nano-particles is $\left(\rho c_{p}\right)_{n f}$ and heat capacitance of the nanofluid is $\left(\rho c_{f}\right)_{n f}$. The ratio of the thermal conductivity to heat capacity of the nano-particles in the fluid is $\alpha_{n f}=\frac{\kappa_{n f}}{\left(\rho c_{p}\right)_{n f}}$. On similar-lines, the ratio of the heat capacitance of the nanofluid with respect to nano-particles is expressed as $\tau_{n f}=\frac{\left(\rho c_{f}\right)_{n f}}{\left(\rho c_{p}\right)_{n f}}$.

\subsection{Non-dimensional form of the equations}

The non-dimensional parameters are introduced as follows:

$r^{\prime}=r / r_{0}, z^{\prime}=z / L_{1}, p^{\prime}=p r_{0}^{2} /\left(u_{0} L_{1} \mu_{f}\right), t^{\prime}=t u_{0} / L_{1}$,

$v^{\prime}=v / u_{0}, \theta=\left(T-T_{1}\right) /\left(T_{0}-T_{1}\right), \sigma=\left(C-C_{1}\right) /\left(C_{0}-C_{1}\right)$, $u^{\prime}=u L_{1} /\left(u_{0} \epsilon\right), \omega=\left(r_{0} \omega / u_{0}\right), J^{\prime}=J / r_{0}^{2}$,

where $r_{0}$ and $u_{0}$ are, respectively, the radii and velocity in the annular region in the absence of tapering and stenosis. $R e=\frac{\rho_{f} u_{0} r_{0}}{\mu_{f}}$ is the Reynolds number. Here, the artery considered for modelling is assumed to be at a distance from the heart and thus the flow is considered as a lowReynolds-number flow.

Introducing the non-dimensional parameters in the governing equations (12)-(17) we get (dropping the prime $\left(^{\prime}\right)$ )

$$
\begin{gathered}
\xi\left(\frac{\partial u}{\partial r}+\frac{u}{r}\right)+\frac{\partial v}{\partial z}=0, \\
\frac{\rho_{n f}}{\rho_{f}} \operatorname{Re} \xi \delta^{3}\left(\xi u \frac{\partial u}{\partial r}+v \frac{\partial u}{\partial z}\right)=-\frac{\partial P}{\partial r}-2 \frac{N}{1-N} C_{\eta} \delta^{2} \frac{\partial \omega}{\partial z} \\
+\left(C_{\mu}+\frac{N}{1-N} C_{\eta}\right) \delta^{2} \xi\left(\frac{\partial^{2} u}{\partial r^{2}}+\frac{1}{r} \frac{\partial u}{\partial r}-\frac{u}{r^{2}}+\delta^{2} \frac{\partial^{2} u}{\partial z^{2}}\right),
\end{gathered}
$$

$$
\begin{aligned}
& \frac{\rho_{n f}}{\rho_{f}} \operatorname{Re} \xi \delta\left(\xi u \frac{\partial v}{\partial r}+v \frac{\partial v}{\partial z}\right)=-\frac{\partial P}{\partial z}+2 \frac{N}{1-N} C_{\eta}\left(\frac{\partial \omega}{\partial r}+\frac{\omega}{r}\right) \\
& \quad+\left(C_{\mu}+\frac{N}{1-N} C_{\eta}\right)\left(\frac{\partial^{2} v}{\partial r^{2}}+\frac{1}{r} \frac{\partial v}{\partial r}\right)+\frac{(\rho \gamma)_{n f}}{(\rho \gamma)_{f}} G_{r} \theta+\frac{(\rho \gamma)_{n f}}{(\rho \gamma)_{f}} B_{r} \sigma
\end{aligned}
$$

$$
\begin{aligned}
& \frac{\rho_{n f}}{\rho_{f}} \operatorname{ReJ} \delta\left(\xi u \frac{\partial \omega}{\partial r}+v \frac{\partial \omega}{\partial z}\right)=2 C_{\eta} \frac{N}{1-N}\left(\delta \frac{\partial v}{\partial z}-\frac{\partial v}{\partial r}\right)-4 \frac{N}{1-N} C_{\eta} \omega \\
& \quad+2 \frac{(2-N)}{M^{2}}\left(\frac{\partial^{2} \omega}{\partial r^{2}}+\delta^{2} \frac{\partial^{2} \omega}{\partial z^{2}}+\frac{1}{r} \frac{\partial \omega}{\partial r}-\frac{\omega}{r^{2}}\right)
\end{aligned}
$$

$$
\begin{aligned}
& \operatorname{RePr} \delta\left(\xi u \frac{\partial \theta}{\partial r}+v \frac{\partial \theta}{\partial z}\right)=\left(\frac{\partial^{2} \theta}{\partial r^{2}}+\frac{1}{r} \frac{\partial \theta}{\partial r}+\delta^{2} \frac{\partial^{2} \theta}{\partial z^{2}}\right) \\
& +N_{b}\left(\frac{\partial \theta}{\partial r} \frac{\partial \sigma}{\partial r}+\delta^{2} \frac{\partial \theta}{\partial z} \frac{\partial \sigma}{\partial z}\right)+N_{t}\left(\left(\frac{\partial \theta}{\partial r}\right)^{2}+\delta^{2}\left(\frac{\partial \theta}{\partial z}\right)^{2}\right)
\end{aligned}
$$

$$
\begin{aligned}
& \operatorname{Re} \operatorname{Pr} \delta\left(\xi u \frac{\partial \sigma}{\partial r}+v \frac{\partial \sigma}{\partial z}\right)=\left(\frac{\partial^{2} \sigma}{\partial r^{2}}+\frac{1}{r} \frac{\partial \sigma}{\partial r}+\delta^{2} \frac{\partial^{2} \sigma}{\partial z^{2}}\right) \\
& +\frac{N_{t}}{N_{b}}\left(\frac{\partial^{2} \theta}{\partial r^{2}}+\frac{1}{r} \frac{\partial \theta}{\partial r}+\delta^{2} \frac{\partial^{2} \theta}{\partial z^{2}}\right) .
\end{aligned}
$$

We consider dynamic viscosity and micro-rotation viscosity of the nanofluid in the form $\mu_{n f}=\mu_{f} C_{\mu}, \eta_{n f}=\eta_{f} C_{\eta}$ where $\mu_{f}$ and $\eta_{f}$ are, respectively, the dynamic viscosity and micro-rotation viscosity of the micro-polar fluid; $C_{\mu}$ and $C_{\eta}$ are functions of nano-particle volume fraction. The micro-polar parameter is denoted by $M^{2}=\frac{r_{0}^{2} \eta_{f}\left[2 \mu_{f}+\eta_{f}\right]}{\Gamma\left[\mu_{f}+\eta_{f}\right]}$ and $N=\frac{\eta_{f}}{\left(\mu_{f}+\eta_{f}\right)}$ is the coupling number.

Under the assumptions of mild stenosis, i.e., $\xi\left(=\epsilon / r_{0}\right)<<1$, and subject to the additional condition $\delta\left(=r_{0} / L_{1}\right) \approx o(1)$, the non-dimensional governing equations (19)-(24) become

$$
\frac{\partial v}{\partial z}=0
$$




$$
\begin{gathered}
\frac{\partial P}{\partial r}=0 \\
\frac{\partial P}{\partial z}=\left(C_{\mu}+\frac{N}{1-N} C_{\eta}\right)\left(\frac{\partial^{2} v}{\partial r^{2}}+\frac{1}{r} \frac{\partial v}{\partial r}\right) \\
+2\left(\frac{N}{1-N}\right) C_{\eta}\left(\frac{\partial \omega}{\partial r}+\frac{\omega}{r}\right) \\
+\beta_{n f} G_{r} \theta+\beta_{n f} B_{r} \sigma \\
\frac{(2-N)}{M^{2}}\left(\frac{\partial^{2} \omega}{\partial r^{2}}+\frac{1}{r} \frac{\partial \omega}{\partial r}-\frac{\omega}{r^{2}}\right)=C_{\eta} \frac{N}{1-N}\left(\frac{\partial v}{\partial r}+2 \omega\right) \\
\left(\frac{1}{r} \frac{\partial}{\partial r}\left(r \frac{\partial \theta}{\partial r}\right)\right)+N_{b}\left(\frac{\partial \sigma}{\partial r} \frac{\partial \theta}{\partial r}\right)+N_{t}\left(\frac{\partial \theta}{\partial r}\right)^{2}=0
\end{gathered}
$$

and

$$
\left(\frac{1}{r} \frac{\partial}{\partial r}\left(r \frac{\partial \sigma}{\partial r}\right)\right)+\frac{N_{t}}{N_{b}}\left(\frac{1}{r} \frac{\partial}{\partial r}\left(r \frac{\partial \theta}{\partial r}\right)\right)=0
$$

where $\beta_{n f}\left(=\frac{(\rho \gamma)_{n f}}{(\rho \gamma)_{f}}\right)$ is the ratio of the thermal expansion coefficients of the nanofluid with respect to the micro-polar fluid, $G_{r}=\frac{r_{0}^{2} g(\rho \gamma)_{f}\left(T_{0}-T_{1}\right)}{u_{0} \mu_{f}}$ is the Grashof number and $B_{r}=$ $\frac{r_{0}^{2} g(\rho \gamma)_{f}\left(C_{0}-C_{1}\right)}{u_{0} \mu_{f}}$ is the solutary Grashof number. $N_{b}=$ $\frac{\left(\rho c_{f}\right)_{n f} D_{B}\left(C_{0}-C_{1}\right)}{\left(\rho c_{p}\right)_{n f}}$ is known as the Brownian motion parameter and $N_{t}=\frac{\left(\rho c_{f}\right)_{n f} D_{T}\left(T_{0}-T_{1}\right)}{T_{1}\left(\rho c_{p}\right)_{n f}}$ is the thermophoresis parameter.

\subsection{Dimensionless boundary conditions}

Endothelium at the vessel wall results in the formation of plaques at one or more parts in the lumen of the artery. This results in reduction of blood flow in the artery. Experiments of Bennett [40] suggest that blood flow is a function of the wall surface, shear stress at the wall and relative cell volumes adjacent to the wall. Chaturani and Biswas [41] conducted a comparative study of polar fluid under various boundary conditions with applications to blood flow.

Hyper-stick condition has been considered at the catheter surface, i.e., the fluid particles neither translate nor rotate at the wall of the catheter. Its mathematical representation is as follows:

$$
v=0 \quad \text { and } \quad \omega=0 \text { at } r=r_{c} .
$$

The effect of boundary layer over arterial surface velocity results in velocity discontinuity and hence the presence of slip at the wall in case of polar fluids is well justified. Therefore

$$
v=u_{1} \quad \text { at } \quad r=h(z) \quad \text { where } \quad L_{0} \leq z \leq L_{0}+L_{1}
$$

also

$v=u_{2} \quad$ at $\quad r=h(z) \quad$ where $\quad z \leq L_{0} \quad$ and $\quad z \geq L_{0}+L_{1}$

where $u_{1}$ and $u_{2}$ are the slip velocities in the stenotic and non-stenotic part of the artery, respectively.

The micro-particles in the continuum will be displaced by the rotation of the particles in the main flow. This is an important phenomenon when fluid is considered as blood or any polymeric suspension. Micro-rotation at the boundary varies with respect to the curl of the velocity vector to the vanishing of micro-rotation. This effect is formulated by introducing the parameter $n_{1}$ as follows:

$$
\omega=-\frac{n_{1}}{2} \frac{\partial v}{\partial r} \quad \text { at } \quad r=h(z), \quad 0 \leq n_{1} \leq 1 .
$$

Boundary conditions for temperature and concentration are defined as follows:

$$
\begin{aligned}
& \theta=1 \quad \text { at } \quad r=r_{c} \text { and } \theta=0 \text { at } r=h(z) ; \\
& \sigma=1 \quad \text { at } \quad r=r_{c} \text { and } \sigma=0 \text { at } r=h(z) .
\end{aligned}
$$

At the catheter surface the drug-coated nano-particle is layered. These boundary conditions help analyse the temperature-sensitive concentration release and its dispersion rate.

\section{Solution process}

The HPM has been used to obtain a perturbation solution to the non-linear and coupled equations (27)-(30).

The basic understanding of the HPM is explained by He [30]. The approximate solution is considered as the sum of an infinite series, which converges rapidly to an accurate solution, which is valid in the domain considered. This technique requires an initial approximation, which is obtained by the original equations satisfying the initial/ boundary conditions.

Consider a non-linear differential equation with boundary conditions as follows:

$$
A(U)=f(r), \quad r \in \Omega ; \quad \text { at } \quad B\left(U, \frac{\partial U}{\partial n}\right)=0, \quad r \in \partial \Omega
$$

where $A$ is a differential operator, which can be separated into linear and non-linear parts:

$$
A=L+N
$$

$f(r)$ is a known analytic function, $B$ is a boundary operator and $\partial \Omega$ is the boundary of the domain $\Omega$. Hence, Eq. (37) can be written as follows: 


$$
\begin{aligned}
& L(U)+N(U)=f(r) \quad r \in \Omega ; \quad \text { at } \quad B\left(U, \frac{\partial U}{\partial n}\right)=0, \\
& \quad r \in \partial \Omega .
\end{aligned}
$$

Define a homotopy that is constructed by Liao [26] as $V(r, q): \Omega \times[0,1] \rightarrow R$, which satisfies

$$
H(q, V)=(1-q)\left(L(V)-L\left(U_{0}\right)\right)+q(L(V)+N(V)-f(r))=0
$$

where $U_{0}$ is the trial solution to the linear operator, satisfying the defined boundary condition.

Equation (39) for the extreme values of the parameter $q$ becomes

$$
\begin{gathered}
H(0, V)=L(V)-L\left(U_{0}\right)=0, \\
H(1, V)=A(V)-f(r)=0 .
\end{gathered}
$$

It is observed that, as the value of embedding parameter $q$ changes from 0 to 1 , the solution $V(r, q)$ varies from $U_{0}(r)$ to $V(r)$. Applying the perturbation technique with the embedding parameter $q \in[0,1]$, the solution in the power series in $q$ is given as

$$
V(r, q)=V_{0}+q V_{1}+q^{2} V_{2}+q^{3} V_{3}+\cdots .
$$

The approximate solution obtained by putting $q=1$ in this equation is

$$
U(r)=\lim _{q \rightarrow 1} V(r, q)=V_{0}+V_{1}+V_{2}+V_{3}+\cdots .
$$

The defined procedure for finding the solution is known as HPM.

\subsection{Implementation of the HPM}

For Eqs. (29) and (30), HPM is used wherein the linear operator is considered as

$$
L=\frac{1}{r} \frac{\partial}{\partial r}\left(r \frac{\partial}{\partial r}\right) .
$$

Therefore

$$
\begin{aligned}
& H(q, \theta)=(1-q)\left[L(\theta)-L\left(\theta_{0}\right)\right] \\
& \quad+q\left[L(\theta)+N_{b}\left(\frac{\partial \sigma}{\partial r} \frac{\partial \sigma}{\partial r}\right)+N_{t}\left(\frac{\partial \theta}{\partial r}\right)^{2}\right]
\end{aligned}
$$

and

$$
\begin{aligned}
& H(q, \sigma)=(1-q)\left[L(\sigma)-L\left(\sigma_{0}\right)\right] \\
& \quad+q\left[L(\sigma)+\frac{N_{t}}{N_{b}}\left(\frac{1}{r} \frac{\partial}{\partial r}\left(r \frac{\partial \theta}{\partial r}\right)\right)\right] .
\end{aligned}
$$

Hence

$$
\begin{aligned}
H(q, \theta)= & \left(\frac{1}{r} \frac{\partial}{\partial r}\left(r \frac{\partial \theta}{\partial r}\right)\right)-\left(\frac{1}{r} \frac{\partial}{\partial r}\left(r \frac{\partial \theta_{0}}{\partial r}\right)\right) \\
& +q\left(\frac{1}{r} \frac{\partial}{\partial r}\left(r \frac{\partial \theta_{0}}{\partial r}\right)\right) \\
& +q\left[N_{b}\left(\frac{\partial \sigma}{\partial r} \frac{\partial \theta}{\partial r}\right)+N_{t}\left(\frac{\partial \theta}{\partial r}\right)^{2}\right] \\
H(q, \sigma)= & \left(\frac{1}{r} \frac{\partial}{\partial r}\left(r \frac{\partial \sigma}{\partial r}\right)\right)-\left(\frac{1}{r} \frac{\partial}{\partial r}\left(r \frac{\partial \sigma_{0}}{\partial r}\right)\right) \\
& +q\left(\frac{1}{r} \frac{\partial}{\partial r}\left(r \frac{\partial \sigma_{0}}{\partial r}\right)\right) \\
& +q\left[\frac{N_{t}}{N_{b}}\left(\frac{1}{r} \frac{\partial}{\partial r}\left(r \frac{\partial \theta}{\partial r}\right)\right)\right]
\end{aligned}
$$

where $q$ is an embedding parameter that has the range $0 \leq q \leq 1$. The homotopies considered for the computation of temperature and concentration variables are as follows:

$$
\begin{gathered}
\theta_{h}(r, q)=\theta_{0}+q \theta_{1}+q^{2} \theta_{2}+q^{3} \theta_{3}+\cdots, \\
\sigma_{h}(r, q)=\sigma_{0}+q \sigma_{1}+q^{2} \sigma_{2}+q^{3} \sigma_{3}+\cdots .
\end{gathered}
$$

Accordingly, the approximate solution of temperature and concentration are given as follows:

$$
\begin{aligned}
& \theta=\lim _{q \rightarrow 1} \theta_{h}=\theta_{0}+\theta_{1}+\theta_{2}+\theta_{3}+\cdots, \\
& \sigma=\lim _{q \rightarrow 1} \sigma_{h}=\sigma_{0}+\sigma_{1}+\sigma_{2}+\sigma_{3}+\cdots
\end{aligned}
$$

Initial approximations $\theta_{0}$ and $\sigma_{0}$ are the solutions of the linear operator w.r.t. $\theta_{h}$ and $\sigma_{h}$ that satisfy the given boundary conditions.

Comparing various powers of $q$ we get the following.

- Zeroth order deformation equations:

$$
\begin{aligned}
& \left(\frac{1}{r} \frac{\partial}{\partial r}\left(r \frac{\partial \theta_{0}}{\partial r}\right)\right)=0 \\
& \left(\frac{1}{r} \frac{\partial}{\partial r}\left(r \frac{\partial \sigma_{0}}{\partial r}\right)\right)=0
\end{aligned}
$$

and the corresponding boundary conditions

$\theta_{0}=1, \sigma_{0}=1$ at $r=r_{c}$ and $\theta_{0}=0, \sigma_{0}=0$ at $r=h(z)$.

Initial guesses with respect to the linear operator are obtained by solving (53) and (54) as

$$
\theta_{0}=\frac{\log (h(z))-\log (r)}{\log (h(z))-\log \left(r_{c}\right)} \quad \text { and } \quad \sigma_{0}=\frac{\log (h(z))-\log (r)}{\log (h(z))-\log \left(r_{c}\right)}
$$


- First-order deformation equations:

$$
\begin{aligned}
& \left(\frac{1}{r} \frac{\partial}{\partial r}\left(r \frac{\partial \theta_{1}}{\partial r}\right)\right)+\left(\frac{1}{r} \frac{\partial}{\partial r}\left(r \frac{\partial \theta_{0}}{\partial r}\right)\right)+N_{b}\left(\frac{\partial \theta_{0}}{\partial r} \frac{\partial \sigma_{0}}{\partial r}\right)+N_{t}\left(\frac{\partial \theta_{0}}{\partial r}\right)^{2}=0 \\
& \left(\frac{1}{r} \frac{\partial}{\partial r}\left(r \frac{\partial \sigma_{1}}{\partial r}\right)\right)+\left(\frac{1}{r} \frac{\partial}{\partial r}\left(r \frac{\partial \sigma_{0}}{\partial r}\right)\right)+\frac{N_{t}}{N_{b}}\left(\frac{1}{r} \frac{\partial}{\partial r}\left(r \frac{\partial \theta_{0}}{\partial r}\right)\right)=0
\end{aligned}
$$

and the corresponding boundary conditions are

$$
\begin{aligned}
& \theta_{1}=0, \quad \sigma_{1}=0 \quad \text { at } \quad r=r_{c} \quad \text { and } \quad \theta_{1}=0 \sigma_{1}=0, \\
& \text { at } \quad r=h(z) .
\end{aligned}
$$

Incorporating the obtained expressions for the temperature and concentration in the coupled non-linear axial velocity and angular momentum equations (27) and (28), we again get coupled non-linear equations. These resultant differential operators are also evaluated using the HPM.

The linear operator for these equations is considered as

$$
L=\frac{1}{r} \frac{\partial}{\partial r}\left(r \frac{\partial}{\partial r}\right)
$$

Therefore

$$
\begin{aligned}
H(q, v)= & (1-q)\left[L(v)-L\left(v_{0}\right)\right] \\
& +q\left[L(v)+\frac{2 N C_{\eta}}{(1-N) C_{\mu}+N C_{\eta}}\left(\frac{\partial \omega}{\partial r}+\frac{\omega}{r}\right)\right. \\
& \left.+\frac{(1-N)}{(1-N) C_{\mu}+N C_{\eta}}\left(\beta_{n f} G_{r} \theta+\beta_{n f} B_{r} \sigma-\frac{\partial P}{\partial z}\right)\right]
\end{aligned}
$$

and

$$
\begin{aligned}
& H(q, \omega)=(1-q)\left[L(\omega)-L\left(\omega_{0}\right)\right] \\
& \quad+q\left[L(\omega)-\frac{C_{\eta} M^{2}}{(2-N)} \frac{N}{1-N}\left(\frac{\partial v}{\partial r}+2 \omega\right)-\frac{\omega}{r^{2}}\right] .
\end{aligned}
$$

The constructions for the axial velocity and micro-rotation equations, respectively, are as follows:

$$
\begin{aligned}
H(q, v)= & \left(\frac{1}{r} \frac{\partial}{\partial r}\left(r \frac{\partial v}{\partial r}\right)\right)-\left(\frac{1}{r} \frac{\partial}{\partial r}\left(r \frac{\partial v_{0}}{\partial r}\right)\right)+q\left(\frac{1}{r} \frac{\partial}{\partial r}\left(r \frac{\partial v_{0}}{\partial r}\right)\right) \\
+ & q\left[\frac{2 N C_{\eta}}{(1-N) C_{\mu}+N C_{\eta}}\left(\frac{\partial \omega}{\partial r}+\frac{\omega}{r}\right)+\frac{(1-N)}{(1-N) C_{\mu}+N C_{\eta}}\right. \\
& \left.\left(\beta_{n f} G_{r} \theta+\beta_{n f} B_{r} \sigma-\frac{\partial P}{\partial z}\right)\right]
\end{aligned}
$$

$$
\begin{aligned}
H(q, \omega)= & \left(\frac{1}{r} \frac{\partial}{\partial r}\left(r \frac{\partial \omega}{\partial r}\right)\right)-\left(\frac{1}{r} \frac{\partial}{\partial r}\left(r \frac{\partial \omega_{0}}{\partial r}\right)\right) \\
& +q\left(\frac{1}{r} \frac{\partial}{\partial r}\left(r \frac{\partial \omega_{0}}{\partial r}\right)\right) \\
& +q\left[-\frac{C_{\eta} M^{2}}{(2-N)} \frac{N}{1-N}\left(\frac{\partial v}{\partial r}+2 \omega\right)-\frac{\omega}{r^{2}}\right]
\end{aligned}
$$

where $q$ is an embedded parameter in the range $0 \leq q \leq 1$. The homotopies considered for the computation of axial and micro-rotational velocity are as follows:

$$
\begin{array}{r}
v_{h}(r, q)=v_{0}+q v_{1}+q^{2} v_{2}+q^{3} v_{3}+\cdots, \\
\omega_{h}(r, q)=\omega_{0}+q \omega_{1}+q^{2} \omega_{2}+q^{3} \omega_{3}+\cdots .
\end{array}
$$

Accordingly, the approximate solutions of axial and microrotational velocity are as follows:

$$
\begin{aligned}
& v=\lim _{q \rightarrow 1} v_{h}(r, q)=v_{0}+v_{1}+v_{2}+v_{3}+\cdots, \\
& \omega=\lim _{q \rightarrow 1} \omega_{h}(r, q) \omega_{0}+\omega_{1}+\omega_{2}+\omega_{3}+\cdots .
\end{aligned}
$$

Comparing the various powers of $q$ leads to the following.

- Zeroth-order deformation equations:

$$
\begin{aligned}
& \left(\frac{1}{r} \frac{\partial}{\partial r}\left(r \frac{\partial v_{0}}{\partial r}\right)\right)=0, \\
& \left(\frac{1}{r} \frac{\partial}{\partial r}\left(r \frac{\partial \omega_{0}}{\partial r}\right)\right)=0 .
\end{aligned}
$$

Corresponding boundary conditions are

$$
\begin{aligned}
& v_{0}=0 \quad \text { and } \quad \omega_{0}=0 \quad \text { at } \quad r=r_{c} \\
& \omega_{0}=-\frac{n_{1}}{2} \frac{\partial v_{0}}{\partial r} \quad \text { at } \quad r=h(z) \\
& v_{0}=\left\{\begin{array}{llc}
u_{1} & \text { at } & r=h(z) \text { where } L_{0} \geq z \quad \text { and } \quad z \geq L_{0}+L_{1}, \\
u_{2} & \text { at } & r=h(z) \text { where } L_{0} \leq z \leq L_{0}+L_{1} .
\end{array}\right.
\end{aligned}
$$

The initial solutions in the non-stenotic region are

$$
\begin{gathered}
v_{0}=u_{1} \frac{\left(\log (r)-\log \left(r_{c}\right)\right)}{\left(\log (h(z))-\log \left(r_{c}\right)\right)}, \\
\omega_{0}=-\frac{n_{1}}{2} \frac{u_{1}}{h(z)} \frac{\left(\log (r)-\log \left(r_{c}\right)\right)}{\left(\log (h(z))-\log \left(r_{c}\right)\right)^{2}} .
\end{gathered}
$$

In the stenotic region they are obtained as

$$
\begin{gathered}
v_{0}(r)=u_{2} \frac{\left(\log (r)-\log \left(r_{c}\right)\right)}{\left(\log (h(z))-\log \left(r_{c}\right)\right)}, \\
\omega_{0}(r)=-\frac{n_{1}}{2} \frac{\left(\log (r)-\log \left(r_{c}\right)\right)}{\left(\log (h(z))-\log \left(r_{c}\right)\right)} .
\end{gathered}
$$

- First-order deformation equations are 


$$
\begin{aligned}
& \left(\frac{1}{r} \frac{\partial}{\partial r}\left(r \frac{\partial v_{1}}{\partial r}\right)\right)+\left(\frac{1}{r} \frac{\partial}{\partial r}\left(r \frac{\partial v_{0}}{\partial r}\right)\right) \\
& \quad+\left(\frac{2 N C_{\eta}}{(1-N) C_{\mu}+N C_{\eta}}\right)\left(\frac{\partial \omega_{0}}{\partial r}+\frac{\omega_{0}}{r}\right) \\
& \quad+\frac{(1-N)}{\left((1-N) C_{\mu}+N C_{\eta}\right)}\left(\beta_{n f} G_{r} \theta_{0}+\beta_{n f} B_{r} \sigma_{0}-\frac{\partial P}{\partial z}\right)=0
\end{aligned}
$$

$$
\begin{aligned}
& \left(\frac{1}{r} \frac{\partial}{\partial r}\left(r \frac{\partial \omega_{1}}{\partial r}\right)\right)+\left(\frac{1}{r} \frac{\partial}{\partial r}\left(r \frac{\partial \omega_{0}}{\partial r}\right)\right) \\
& \quad-\frac{C_{\eta} M^{2}}{(2-N)} \frac{N}{(1-N)}\left(\frac{\partial v_{0}}{\partial r}+2 \omega_{0}\right)-\frac{\omega_{0}}{r^{2}}=0 .
\end{aligned}
$$

Corresponding boundary conditions are

$$
\begin{aligned}
& v_{1}=0 \quad \text { and } \quad \omega_{1}=0 \quad \text { at } \quad r=r_{c}, \\
& \omega_{1}=-\frac{n_{1}}{2} \frac{\partial v_{1}}{\partial r} \text { at } r=h(z), \\
& v_{1}=\left\{\begin{array}{lll}
0 & \text { at } \quad r=h(z) \text { where } L_{0} \geq z \text { and } z \geq L_{0}+L_{1}, \\
0 & \text { at } \quad r=h(z) \text { where } L_{0} \leq z \leq L_{0}+L_{1} .
\end{array}\right.
\end{aligned}
$$

\subsection{Evaluation of Nusselt number and Sherwood number}

The ratio of conductive heat transfer to the convective heat transfer across the boundary is known as the Nusselt number $(\mathrm{Nu})$ and is given by the relation

$$
N u=\frac{\left.\frac{\partial\left(T-T_{0}\right)}{\partial r}\right|_{r=r_{c}}}{\frac{\left(T_{1}-T_{0}\right)}{r_{0}}}
$$

Nusselt number expression in the non-dimensional form is given as

$$
N u=-\left.\left(\frac{\partial \theta}{\partial r}\right)\right|_{r=r_{c}} .
$$

In the mass transfer phenomenon, the Sherwood number $(S h)$ is the mass transfer Nusselt number, which is the ratio of the convective mass transfer to the rate of diffusive mass transport and is given by the

\begin{tabular}{|c|c|c|c|}
\hline \multirow[b]{2}{*}{$r$} & \multicolumn{2}{|c|}{ Asymmetric stenosis } & \multirow[b]{2}{*}{$\begin{array}{c}\text { The present } \\
\text { model with } \omega \text { - } \\
\text { shaped stenosis } \\
\text { HPM }\end{array}$} \\
\hline & $\begin{array}{l}\text { Analytical } \\
\text { given by } \\
\text { Elnaqeeb } \\
\text { et al }[31]\end{array}$ & $\begin{array}{c}\text { Implementation of } \\
\text { HPM for Elnaqeeb } \\
\text { et al }[31] \text { modelled } \\
\text { problem }\end{array}$ & \\
\hline 0.06 & 1.00000 & 1.00000 & 1.0000 \\
\hline 0.07175 & 0.94319816 & 0.94295361 & 0.98794563 \\
\hline 0.011875 & 0.77784196 & 0.77769732 & 0.94089734 \\
\hline 0.248 & 0.52549189 & 0.526943283 & 0.804989572 \\
\hline 0.3185 & 0.43721500 & 0.439033292 & 0.73051233 \\
\hline 0.37725 & 0.37662290 & 0.378507053 & 0.668928683 \\
\hline 0.4125 & 0.34431559 & 0.346166845 & 0.631997752 \\
\hline 0.51825 & 0.26046607 & 0.262029125 & 0.520551345 \\
\hline 0.61225 & 0.19769269 & 0.198895383 & 0.420268841 \\
\hline 0.671 & 0.162462825 & 0.163434395 & 0.357046565 \\
\hline 0.718 & 0.136034202 & 0.1368281 & 0.306238196 \\
\hline 0.77675 & 0.104839264 & 0.105426247 & 0.242527902 \\
\hline 0.8355 & 0.075377945 & 0.075778707 & 0.178691493 \\
\hline 0.89425 & 0.047380339 & 0.04761748 & 0.114820983 \\
\hline 0.9295 & 0.031191612 & 0.031341614 & 0.076514728 \\
\hline 0.98825 & 0.005097507 & 0.005120314 & 0.012740762 \\
\hline 1 & 0 & 0 & 0 \\
\hline
\end{tabular}
relation

$$
S h=\frac{\left.\frac{\partial\left(C-C_{0}\right)}{\partial r}\right|_{r=r_{c}}}{\frac{\left(C_{1}-C_{0}\right)}{r_{0}}}
$$

Sherwood number expression in the non-dimensional form is given as

$$
S h=-\left.\left(\frac{\partial \sigma}{\partial r}\right)\right|_{r=r_{c}}
$$

Table 2. Comparison of the present results with the exiting results.

\section{Results and discussion}

The present study is dedicated to understanding the rheological properties of blood flow through $\omega$-shaped stenotic tapered artery in the presence of a catheter. The blood is modelled as silver nano-particles immersed in a micropolar fluid and HPM is used to obtain asymptotic solutions to the modelled nonlinear coupled equations. The effects of parameters like tapering angle, thermophoresis parameter and Brownian motion parameter on the dispersion of temperature and concentration are studied. Profiles of axial and micro-rotation velocity have been discussed for various values of nano-particle volume fraction. Further, the effects of various parameters arising out of the geometry and fluid considered on physiological flow properties such as the resistance of the flow and the wall shear stress are also computed and analysed. The fixed values of various parameters used in the computation are given as follows: $r_{0}=0.152, L=2, L_{1}=1, L_{0}=0.5, u_{1}=1, u_{2}=2, \quad M=$ $1, N=0.1, N_{b}=2, N_{t}=0.7, G_{r}=2, B_{r}=1, \zeta=0.05, \epsilon=$ 0.05 and $r_{c}=0.05$. The following are the investigation in detail.

In order to validate the scheme adopted, the analytical results obtained by Elnaqeeb et al [31] for temperature of viscous fluid flow are compared with the results obtained by us using HPM for the model discussed by Elnaqeeb et al [31] and for the model considered in the present 
problem and are displayed in table 2. It is observed that the results obtained using the analytical method and the HPM method match up to three decimal points. Therefore, it can be understood that the present method is well suited for this kind of modelled equations, thus validating the method used. Further, the results obtained for the present model as shown in column three of table 2 are on similar lines to the first and second column results. This validates our approach for the present model. Further, the temperature dispersion is more when compared with the results of Elnaqeeb. This highlights the valuableness of the present model in terms of the geometry and fluid considered along with the associated boundary conditions, as higher dispersion results in better drug delivery.

\subsection{Temperature and concentration}

In order to understand the convergence of the present method, the temperature profiles for various orders of deformation are depicted in figure 2. Here, it is observed that the curves converge from the third-order deformation onwards. Hence, in the present analysis, up to fourth-order deformation is considered. Understanding the impact of tapering angle and height of the stenosis on the temperature and concentration dispersion is carried out through figures 5-8. In particular, the effect of height of stenosis on these profiles is observed through figures 5 and 6 , wherein it is seen that as the height of the stenosis increases, both the temperature and concentration profiles decrease. Further, it can be concluded that the dispersion rate is more for the mild stenosis when compared with that of the severe stenosis. Hence, this technique performs better in the starting stage of the stenosis.

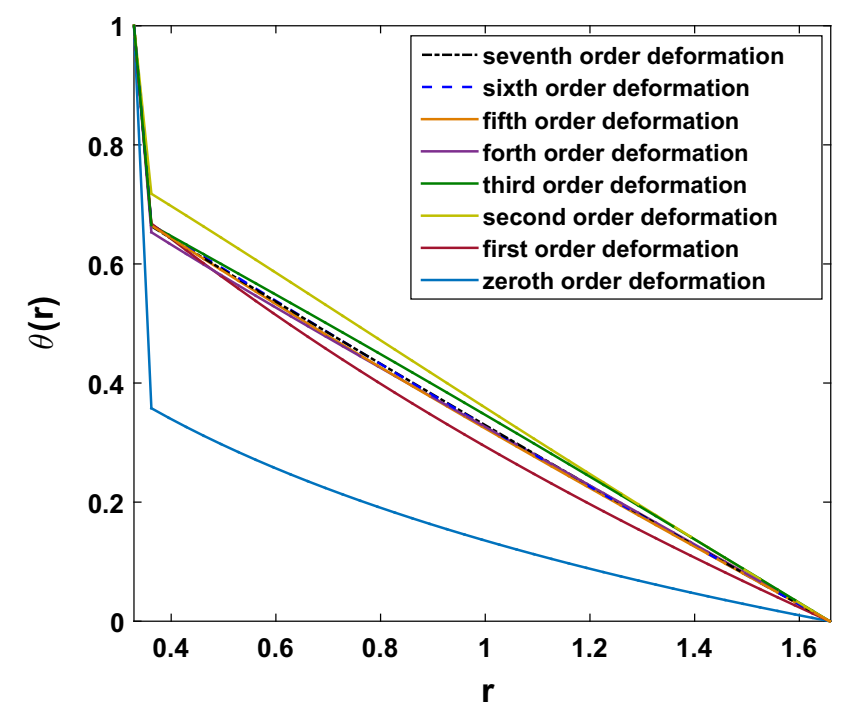

Figure 2. Temperature variation in the radial direction for different orders of deformation at $z=0.9$.

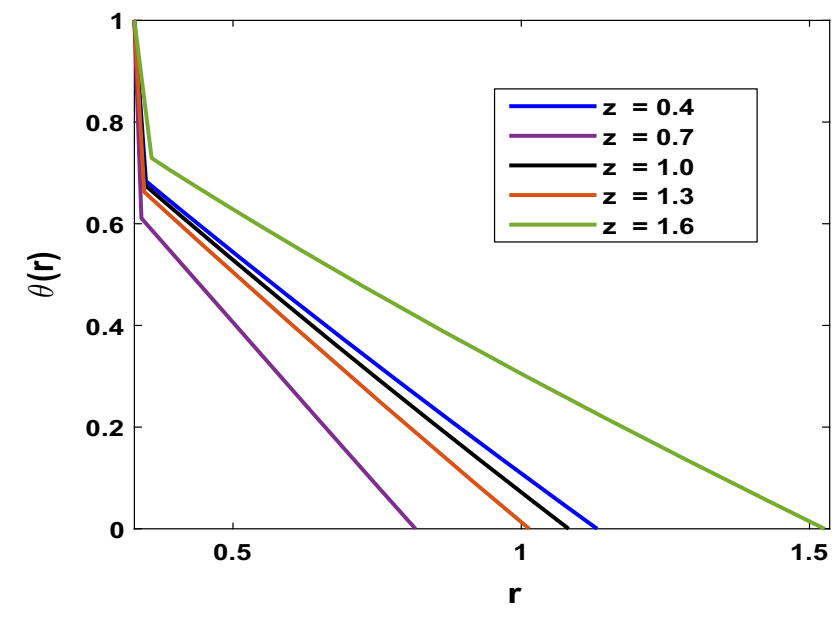

Figure 3. Temperature variation with $r$ at different values of $z$.

Further, the result of temperature and concentration variation for different tapering angles is as shown in figures 7 and 8, respectively. As the artery tapers from convergence to divergence, the temperature and concentration profiles increase at all axial sections. From these figures, it can be seen that the high dispersion results occur in the diverging artery due to the high Brownian motion of the particles. Thus, these dispersion results are in line with the wet diameter. Having understood the effect of the tapering angle, all further results are computed for the diverging tapered artery. Figure 3 depicts the behaviour of temperature variation form the catheter surface to the arterial surface at different locations in and around the stenotic region. It can be seen that the temperature values are more in the non-stenotic region when compared with that of stenotic region, with the maximum values observed in the poststenotic region. However, the dispersion is more in the

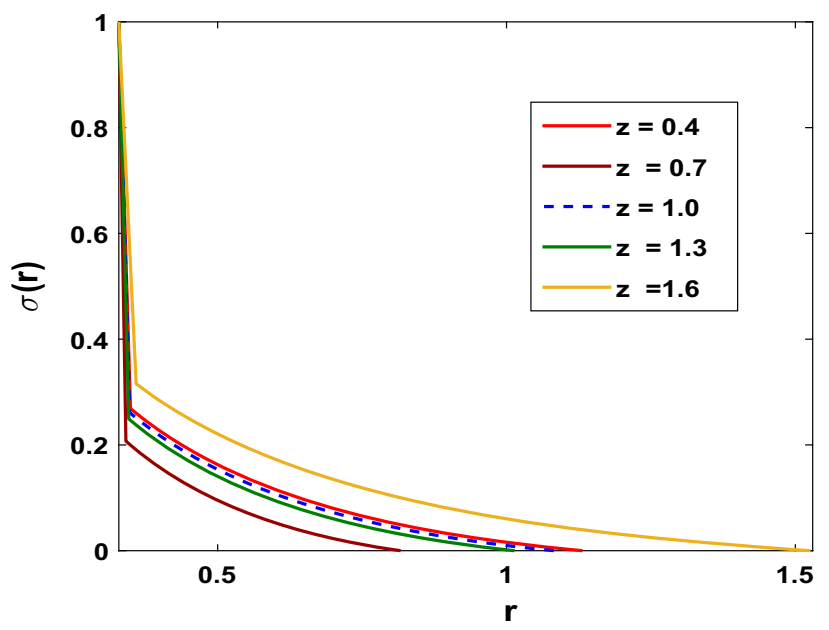

Figure 4. Concentration variation with $r$ at different values of $z$. 


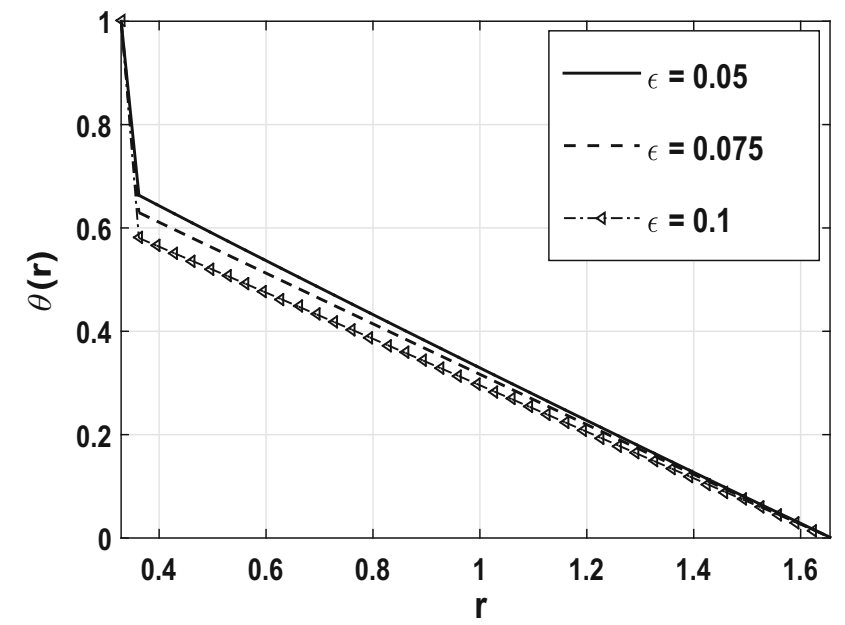

Figure 5. Temperature distribution with different heights of stenosis at $z=0.9$.

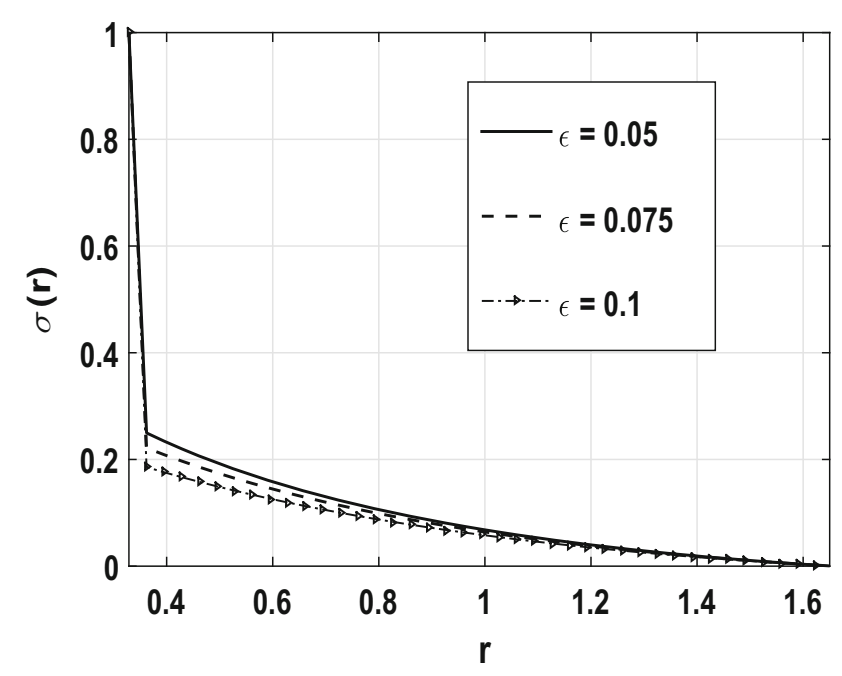

Figure 6. Concentration distribution with different heights of stenosis at $z=0.9$.

stenotic region and it is maximum at the first extremum followed by the second extremum.

Further variation of the concentration from the catheter wall to arterial wall is shown in figure 4. Here, it is observed that the drug concentration is more at the catheter wall and approaches zero at the arterial surface. It can be concluded that the drug dispersion is more at the extremes of the stenosis and it is maximum at the location corresponding to the first extremum. Hence, this process of drug delivery using the catheter is more durable in the treatment of the obstruction of the artery (as happens in CVDs) with less side effects due to high heat and mass transfer at the extremes.

4.1a Effects of $N_{b}$ and $N_{t}$ : The impact of the Brownian motion parameter $N_{b}$ and thermophoresis parameter $N_{t}$ on the temperature and concentration distribution is shown in

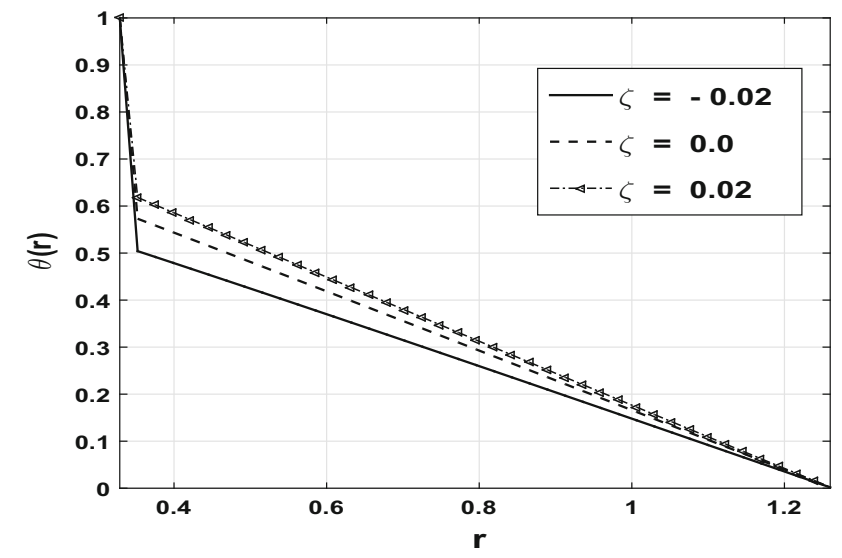

Figure 7. Temperature distribution with different values of taper angle at $z=0.9$.

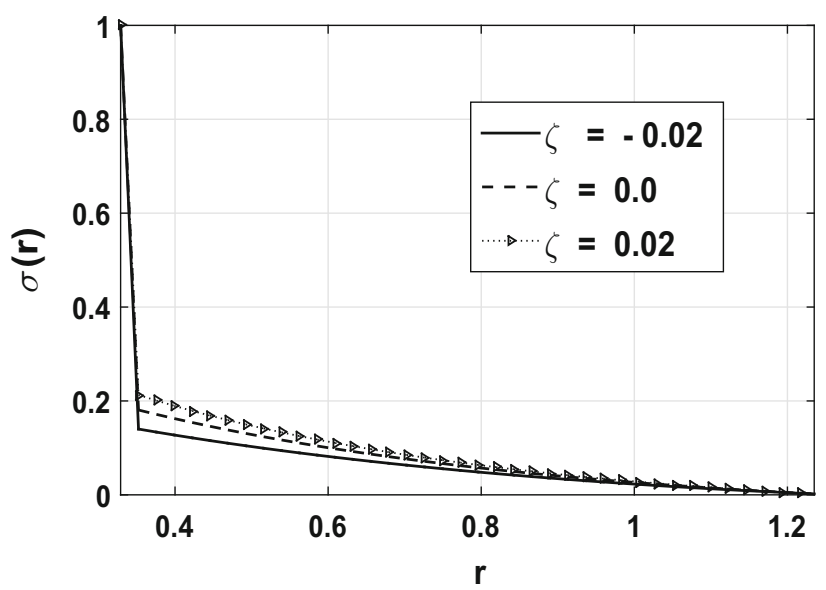

Figure 8. Concentration distribution with different values of taper angle at $z=0.9$.

figures 9-12. From figures 9 and 10, it is observed that the increase in the Brownian motion parameter $N_{b}$ results in increase in the temperature and concentration profiles. The rise in Brownian motion parameter leads to the bombardment of the molecules with the surrounding fluid, which increases the erratic random movement of microscopic particles in the fluid, thus resulting in the increase of temperature and concentration values. Further, it can be concluded that the corresponding dispersion is high in case of Brownian motion parameter.

The variation in thermophoresis parameter $N_{t}$ is directly proportional to the temperature and hence temperature dispersion, whereas it is inversely related to the concentration and hence concentration dispersion; the same is depicted in figures 11 and 12, respectively. This is because there is a temperature difference between the drug-coated catheter and the surrounding medium. Under the action of such temperature difference, the particles gain heat and 


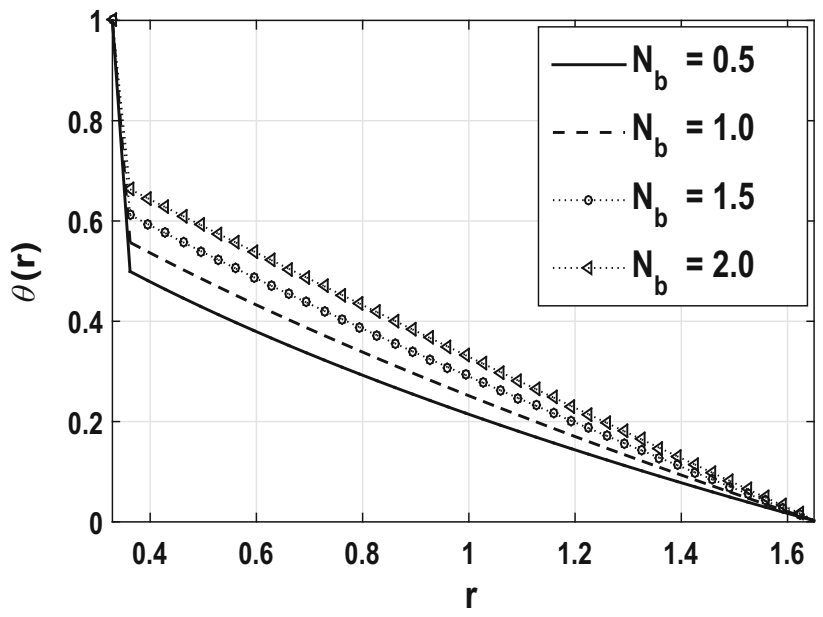

Figure 9. Effect of $N_{b}$ on temperature.

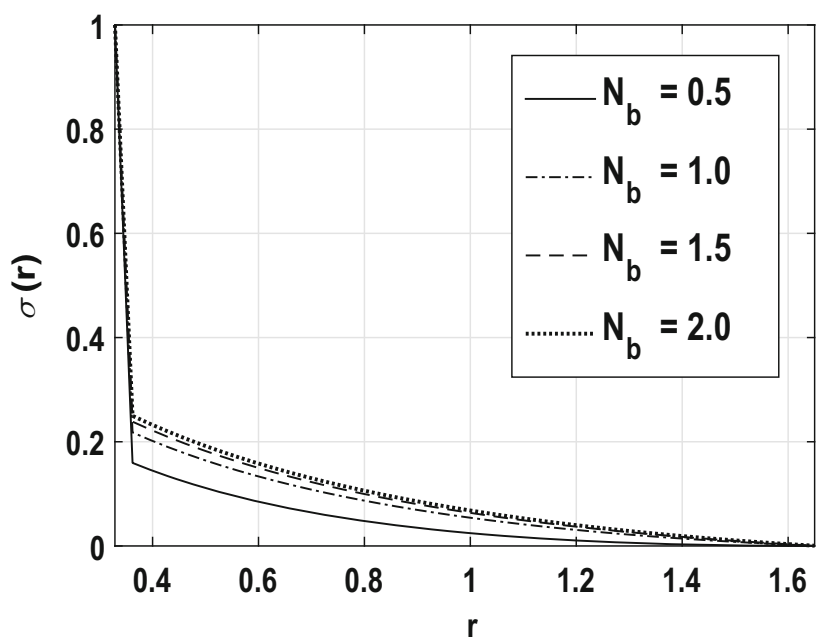

Figure 10. Effect of $N_{b}$ on concentration.

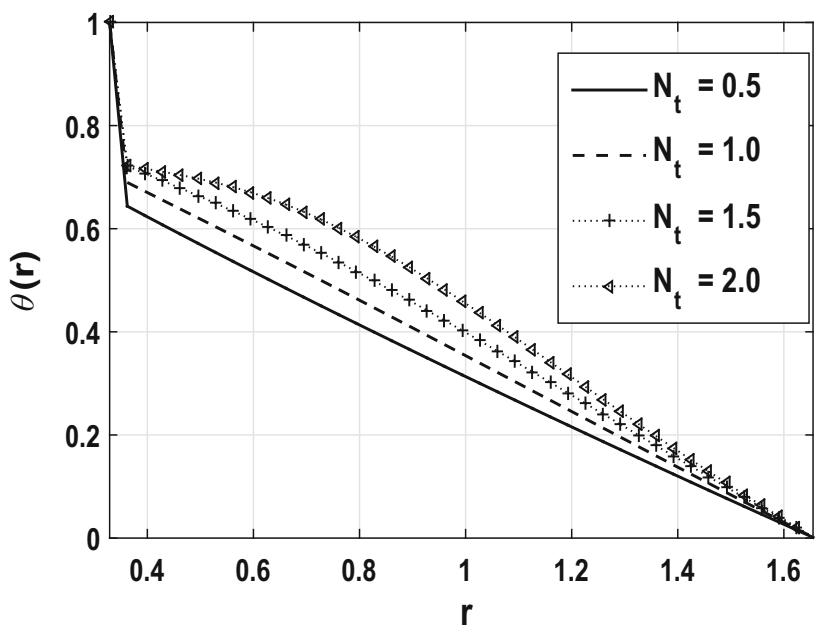

Figure 11. Effect of $N_{t}$ on temperature.

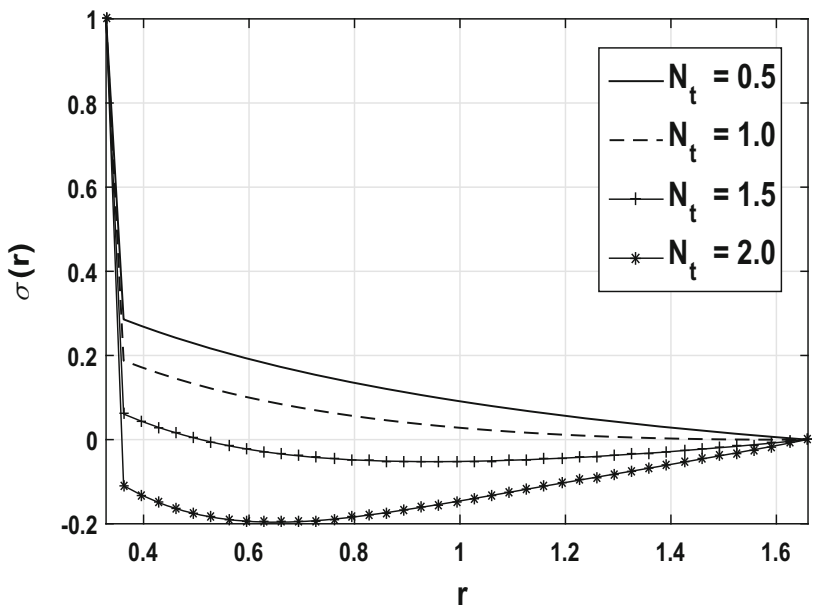

Figure 12. Effect of $N_{t}$ on concentration.

move away from the catheter surface along with the drug. After certain threshold value of $N_{t}$, an erratic change in temperature and concentration dispersion is observed due to the effects of thermal conductivity and drug discharge from silver nano-particles. This results in the increase of temperature dispersion when $N_{t}$ increases. However, the concentration dispersion approaches even negative values when $N_{t}$ increases. It is to be noted that the phenomenon of the nano-particle increases as the Brownian motion parameter increases while the reverse trend is seen with the increase in the thermophoresis parameter. Hence we see opposite behaviour for the concentration profiles in case of these two parameters.

The dependences of $N u$ and $S h$ on thermophoresis parameter $N_{b}$ and Brownian motion parameter $N_{t}$ are shown in the form of tables 3 and 4 . As $N_{b}$ increases, the average Nusselt number and average Sherwood number also increase. Similarly, as $N_{t}$ increases, the average Nusselt number increases, but the trend is reversed in case of the

Table 3. $\overline{N u}$ and $\overline{S h}$ for different $N_{b}$ and $N_{t}=0.7$.

\begin{tabular}{ccccc}
\hline & & $N_{t}$ & $N u$ & $S h$ \\
\hline$N_{b}$ & 0.5 & 0.7 & 0.68111 & 0.24231 \\
& 0.7 & & 0.71090 & 0.28562 \\
& 1.0 & & 0.75450 & 0.31881 \\
& 1.5 & & 0.82337 & 0.34613 \\
\hline
\end{tabular}

Table 4. $\overline{N u}$ and $\overline{S h}$ for different $N_{t}$ and $N_{b}=2.0$.

\begin{tabular}{ccccc}
\hline & & $N_{b}$ & $N u$ & $S h$ \\
\hline$N_{t}$ & 0.1 & 2.0 & 0.81051 & 0.48256 \\
& 0.5 & & 0.86249 & 0.40673 \\
& 1.0 & & 0.91828 & 0.28410 \\
& 1.5 & & 0.95322 & 0.13158 \\
\hline
\end{tabular}


average Sherwood number. This is justified as $N u$ is dependent on temperature while $S h$ is dependent on concentration.

\subsection{Velocity}

The velocity components in the arterial domain are investigated through figures 13-18. The variation of the axial velocity in the stenotic region corresponding to $z=0.9$ has been discussed for different nano-particles (Aluminium Oxide nano-particles, Titanium Oxide nano-particles and Copper nano-particles) in case of the diverging tapered artery as shown in figure 13 . Here, radius is assumed to vary from the catheter surface to the arterial surface. The details of the thermo-physical properties required to evaluate the velocity profiles for various nano-particles are recorded in the form of table 1. From the graph, it is noticed that the immersion of silver nano-particles in the micro-polar fluid enhances the change in velocity more as

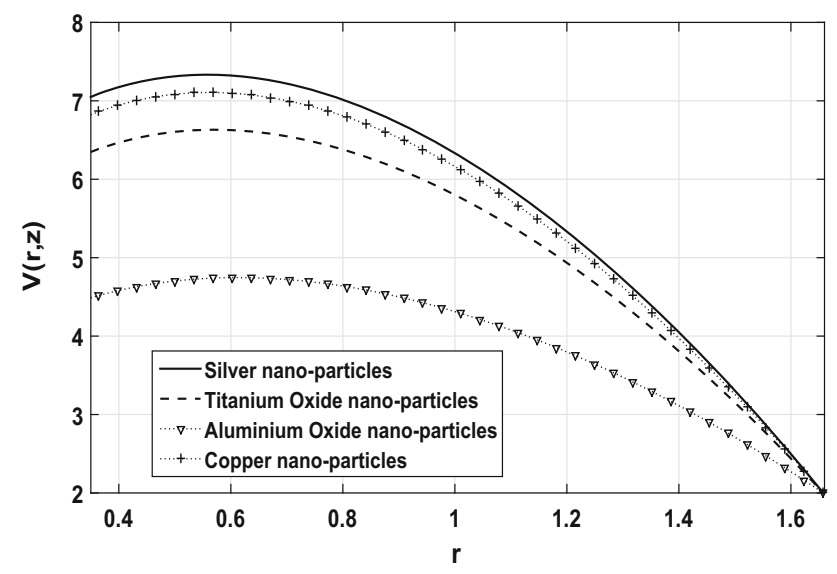

Figure 13. Axial velocity variation with several nanofluids.

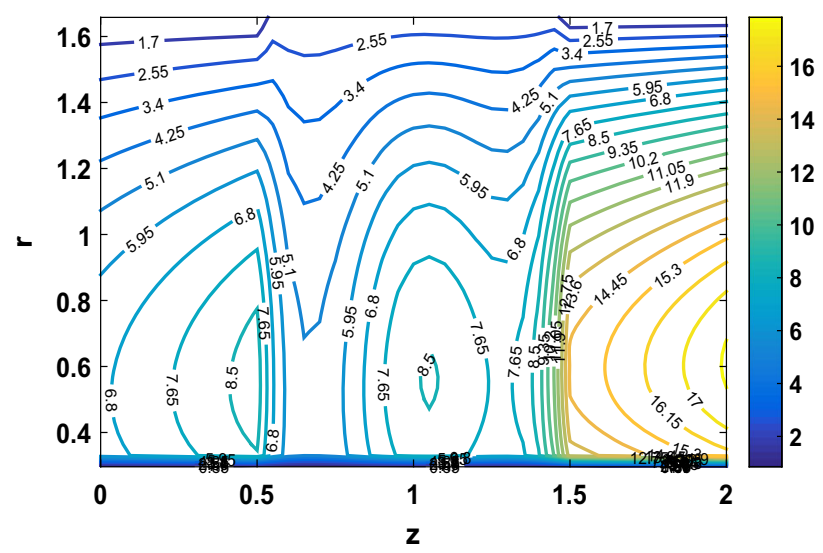

Figure 14. Axial velocity contour in axial and radial directions with $4 \%$ nano-particle volume fraction.

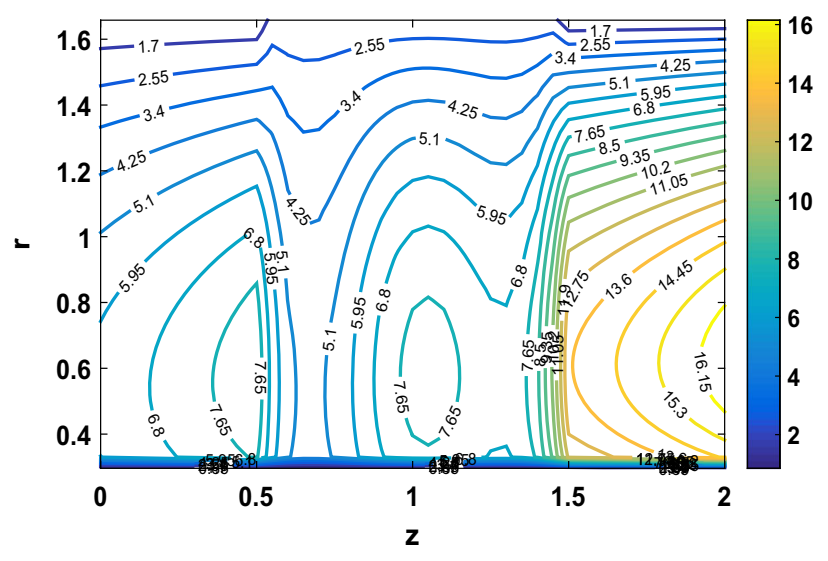

Figure 15. Axial velocity contour in axial and radial directions with $0 \%$ nano-particle volume fraction.

compared with the immersion of other (Aluminium Oxide nano-particles, Titanium Oxide nano-particles and Copper nano-particles) nano-particles. This leads to the higher transfer rate of heat/concentration in the considered stenotic arterial segment. Therefore, to enhance the drug dispersion and drug delivery to the targeted organs, silver nano-particles are considered in the computation of flow variables. The change in the volume fraction of the immersed nanoparticles causes the change in density, viscosity and fluid properties, which leads to the alteration in flow dynamics of the rheology. Therefore, the change in axial velocity and micro-rotation velocity with respect to the volume fraction is discussed in detail. The axial velocity contours are computed for $4 \%$ and $0 \%$ of volume fraction and are depicted in figures 14 and 15 , respectively. It is noticed that in diverging tapered artery, the axial velocity is maximum in the post-stenotic region and minimum in the pre-stenotic region for a given $r$. It is worth mentioning that a secondary flow is observed in the stenotic region. It is to be mentioned that for a given $r$ the velocity slightly decreases when the fluid is entering the stenosis, i.e., when $z$ is around 0.5 , and there is a slight arrest of the fluid for the same range of $z$ near the arterial wall. Further, one can see that an increase in the volume fraction leads to the increase in the axial velocity for a given $r$ and $z$. Also, a strong secondary flow is observed in the stenotic region, for higher volume fraction as compared with that of lower volume fraction. Hence, it can be concluded that the higher volume fraction leads to higher shear stress. In general, high intensity in velocity is observed in the post-stenotic region as compared with the pre-stenotic region, which is responsible for generating the turbulence in the flow even for small Reynolds number; this is responsible for the "arterial murmurs".

The micro-rotation velocity contours are shown in figures 17 and 18 in case of diverging tapered artery for $4 \%$ and $0 \%$ nano-particle volume fraction, respectively. In general, it is noticed that the intensity of the micro-rotation 
velocity is much higher and it has separable flow behaviour in case of higher nano-particle volume fraction. A strong flow separation with much higher intensity is observed in the immediate post-stenotic region when compared with that of the separated flow between the two extrema of the stenosis. It is found out that the presence of higher slip in the stenotic portion results in higher micro-rotation velocity.

4.2a Impedance and wall shear stress: The pressure drop, volumetric flow, impedance and shear stress are derived from the fundamental flow properties. The effects of the pressure drop, impedance and shear stress at the wall are computed and depicted in figures 16, 19 and 20, respectively. From figure 16, it is observed that the axial pressure is high in the main flow region and it decreases in the peripheral region. In case of diverging tapered artery, the axial pressure difference is less at the first extremum of the stenosis as compared with the last extremum of the

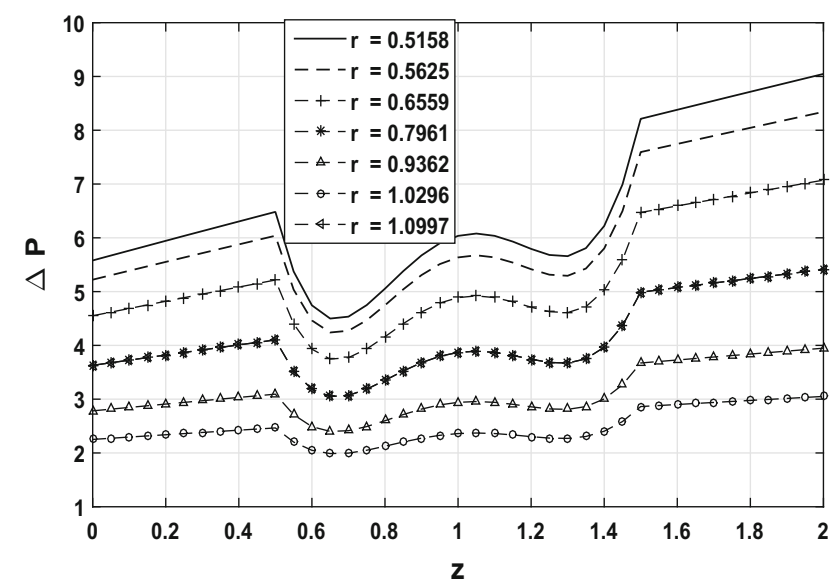

Figure 16. Variation of pressure along the axial direction for different annular sections.

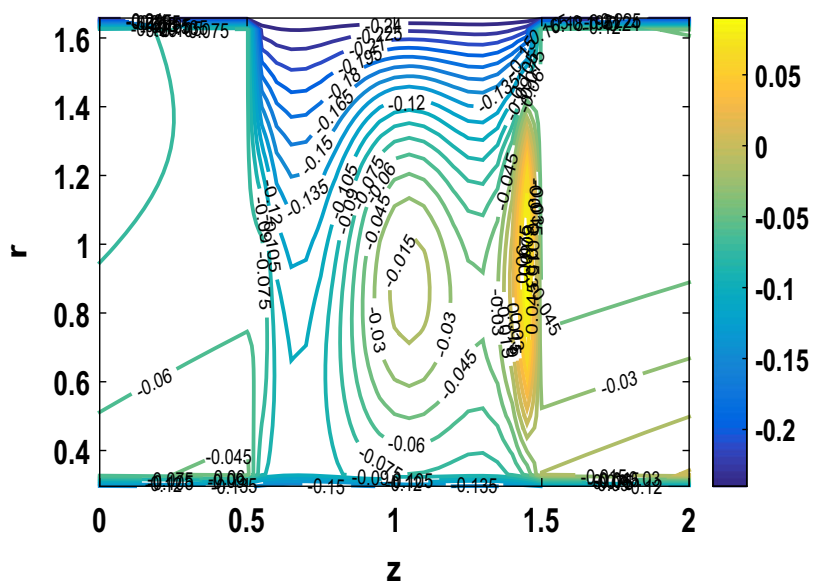

Figure 17. Micro-rotation velocity contour in axial and radial directions with $4 \%$ nano-particle volume fraction.

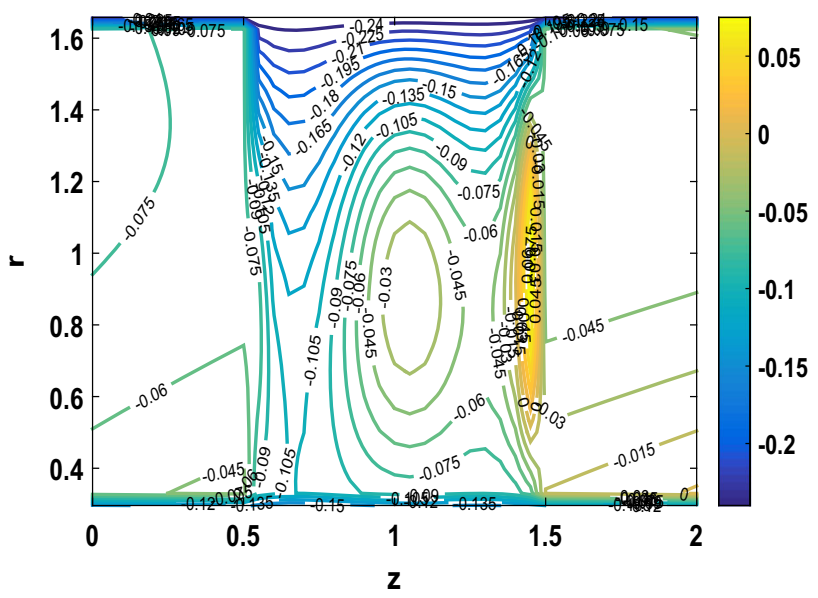

Figure 18. Micro-rotation velocity contour in axial and radial directions with $4 \%$ nano-particle volume fraction.

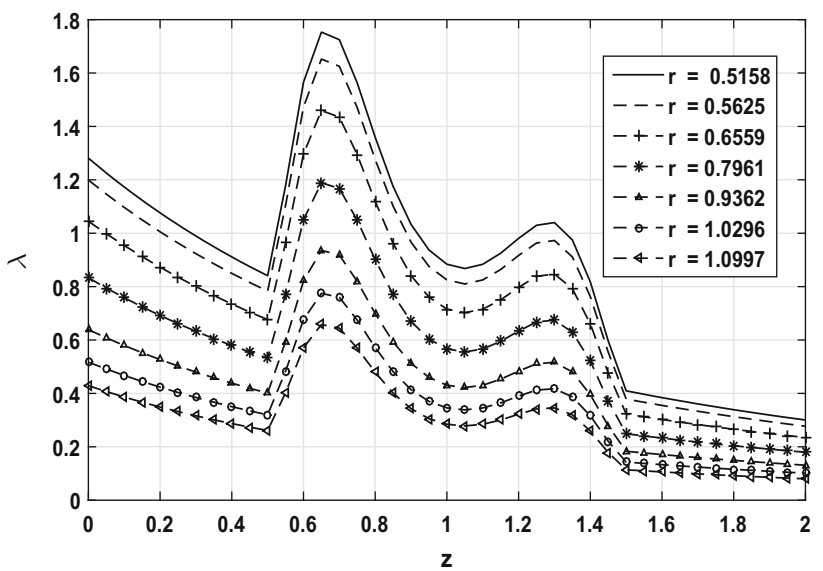

Figure 19. Variation of impedance across the axial direction for different annular sections.

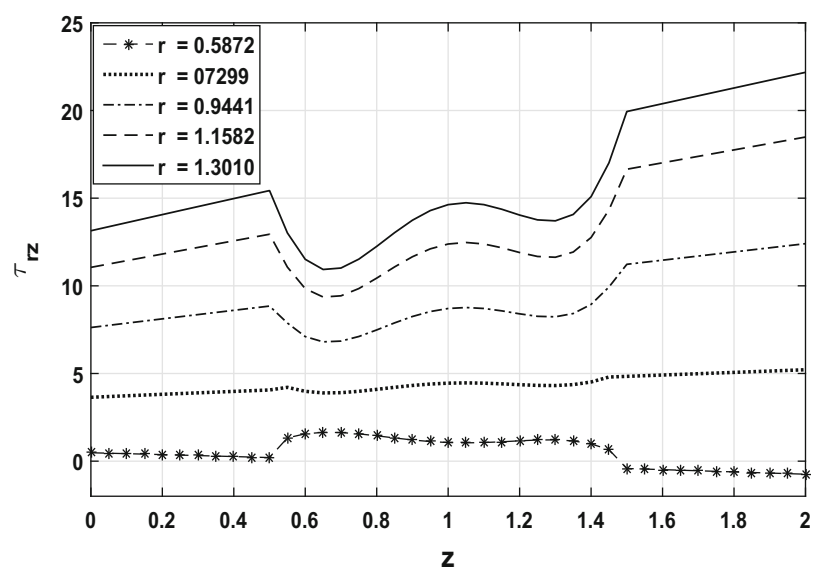

Figure 20. Variation of shear stress along the axial direction for different annular sections. 
stenosis. A similar effect is observed in the volumetric flow rate; however, the intensity is maximum as compared with the pressure difference. One of the important physiological parameters called resistance to the flow is calculated as the fraction of pressure difference versus volumetric flow in the arterial segment. It is noticed that the volumetric flow rate dominates the pressure drop in the stenotic region, whereas the dominance is quantitatively less in the non-stenotic region. The same can be understood from figure 19. High impedance is observed in the first extremum of the stenosis compared with the second extremum of the $\omega$-shaped stenosis. This is due to the change in the annular region caused by the diverging nature of the tapered artery. The variation in the axial direction of the other important physiological property called wall shear stress is studied for different annular region as shown in figure 20. Here, it is observed that the wall shear stress in the stenotic region is less as compared with the non-stenotic region. This is due to the low velocity in the stenotic region as compared with the non-stenotic region. The same can be understood from figure 14. It is found out that negative shear stress is observed in the post-stenotic region near the catheter surface. This is due to the high velocity in the pre-stenotic and stenotic regions as compared with the post-stenotic region.

\section{Summary and conclusions}

In this article, the authors considered blood flow through a stenotic tapered artery to treat the stenotic conditions. The temperature-sensitive drug is coated on nano-particles and is layered on the outer surface of the catheter. The physical model is constructed such that the required temperature for drug release is supplied through the catheter. The rheology of the blood is considered to be that of a polar fluid, in particular a micropolar fluid with the inclusion of nanoparticles in the fluid. The HPM is successfully implemented to analyse the dynamics of the blood flow along with the dispersion of temperature and concentration. The highlights of the investigation is summarized in detail as follows:

1. The convergence of the HPM method is obtained from the fourth-order deformation, as shown in figure 2.

2. The obtained HPM results are validated with the analytical results presented by Elnaqeeb et al [31] by comparing the temperature values. The details are presented in the description of table 2. The results obtained using the present method are in excellent agreement to the existing result.

3. The increase in stenosis height leads to decrease in temperature and concentration profiles, whereas temperature and concentration profiles increase for increase in tapering parameter. Further, high dispersion rate is noticed in case of mild stenosis.

4. It is observed that the temperature and drug concentration dispersion are more at the extremes of the stenosis with the maximum being at the location corresponding to the first extremum.

5. It observed that the rise in Brownian motion parameter leads to increase in the erratic random movement of microscopic particles in the fluid, thus resulting in the increase of the temperature and concentration values.

6. It is observed that silver nano-particles enhance the change in velocity more as compared with that of other nano-particles considered in the problem, thus leading to higher transfer rate in the affected region.

7. The nano-particle volume fraction $(\phi)$ is effectively studied on axial velocity and microrotation velocity. The velocity is more in the post-stenotic region and it is the least in the pre-stenotic region for a given $r$ with secondary flow in the stenotic region. Further, high volume fraction leads to occurrence of strong secondary flow in the stenotic region.

8. It is noticed that, the volumetric flow rate dominates the pressure drop in the stenotic region, whereas the dominance is quantitatively less in the non-stenotic region.

9. The wall shear stress in the stenotic region is less as compared with the non-stenotic region, due to the low velocity in the stenotic region as compared with the nonstenotic region.

This theoretical study will act as a prototype in biomedicine for the cure of vessel vascular diseases through DrugCoated Balloon (DCB) angioplasty.

\section{Compliance with ethical standards}

Conflict of interest On behalf of all the authors, the corresponding author states that there is no conflict of interest.

\section{References}

[1] World Health Organization 2016 World health statistics 2016: monitoring health for the SDGs (sustainable development goals). World Health Organization

[2] Gupta R, Joshi P, Mohan V, Reddy K S and Yusuf S 2008 Epidemiology and causation of coronary heart disease and stroke in India. Heart 94(1): 16-26

[3] Gupta R, Mohan I and Narula J 2016 Trends in coronary heart disease epidemiology in India. Annals of Global Health 82(2): 307-315

[4] Fung Y C 1971 Biomechanics: a survey of the blood flow problem. Advances in Applied Mechanics 11: 65-130

[5] Young D F and Tsai F Y 1973 Flow characteristics in models of arterial stenoses ii: unsteady flow. Journal of Biomechanics 6(5): 547-559

[6] Bluestein D, Niu L, Schoephoerster R T and Dewanjee M K 1997 Fluid mechanics of arterial stenosis: relationship to the development of mural thrombus. Annals of Biomedical Engineering 25(2): 344-356

[7] Ariman T, Turk M A and Sylvester N D 1973 Microcontinuum fluid mechanics-a review. International Journal of Engineering Science 11(8): 905-930 
[8] Schmid-Schönbein G W, Usami S, Skalak R and Chien S 1980 The interaction of leukocytes and erythrocytes in capillary and postcapillary vessels. Microvascular Research 19(1): 45-70

[9] Reddy J V R, Srikanth D and Murthy S K 2014 Mathematical modelling of couple stresses on fluid flow in constricted tapered artery in presence of slip velocity-effects of catheter. Applied Mathematics and Mechanics 35(8): 947-958

[10] Agrawal V, Paul C, Das M K and Muralidhar K 2015 Effect of coil embolization on blood flow through a saccular cerebral aneurysm. Sadhana 40(3): 875-887

[11] Priyadharshini S and Ponalagusamy R 2017 Computational model on pulsatile flow of blood through a tapered arterial stenosis with radially variable viscosity and magnetic field. Sādhanā 42(11): 1901-1913

[12] Cemal Eringen A 1964 Simple microfluids. International Journal of Engineering Science 2(2): 205-217

[13] Ramana Reddy J V, Srikanth D and Krishna Murthy S V S S N V G 2016 Mathematical modelling of time dependent flow of non-newtonian fluid through unsymmetric stenotic tapered artery: effects of catheter and slip velocity. Meccanica 51(1): 55-69

[14] Mekheimer K S and El Kot M A 2008 The micropolar fluid model for blood flow through a tapered artery with a stenosis. Acta Mechanica Sinica 24(6): 637-644

[15] Roos R and Lykoudis P S 1971 The fluid mechanics of the ureter. Journal of Fluid Mechanics 46(04): 625-630

[16] MacDonald D A 1986 Pulsatile flow in a catheterised artery. Journal of Biomechanics 19(3): 239-249

[17] Dash R K, Jayaraman G and Mehta K N 1999 Flow in a catheterized curved artery with stenosis. Journal of Biomechanics 32(1): 49-61

[18] Srinivasacharya D and Srikanth D 2012 Flow of micropolar fluid through catheterized artery-a mathematical model. International Journal of Biomathematics 5(02): 1250019

[19] Reddy J V and Srikanth D 2015 The polar fluid model for blood flow through a tapered artery with overlapping stenosis: effects of catheter and velocity slip. Applied Bionics and Biomechanics 2015 https://doi.org/10.1155/2015/174387

[20] Mekheimer K S, Haroun M H and El Kot M A 2012 Influence of heat and chemical reactions on blood flow through an anisotropically tapered elastic arteries with overlapping stenosis. Applied Mathematics and Information Sciences 6(2): 281-292

[21] Mekheimer K S and El Kot M A 2012 Mathematical modeling of axial flow between two eccentric cylinders: application on the injection of eccentric catheter through stenotic arteries. International Journal of Non-Linear Mechanics 47(8): 927-937

[22] Mekheimer K S, Salama F and El Kot MA 2014 The unsteady flow of a Carreau fluid through inclined catheterized arteries having a balloon with time-variant overlapping stenosis. Walailak Journal of Science and Technology 12(10): 863-883

[23] Mekheimer K S and El Kot M A 2015 Suspension model for blood flow through catheterized curved artery with timevariant overlapping stenosis. Engineering Science and Technology 18(3): 452-462
[24] Chol S U S 1995 Enhancing thermal conductivity of fluids with nanoparticles. ASME-Publications-FED 231: 99-106

[25] Yu W, France D M, Routbort J L and Choi S U S 2008 Review and comparison of nanofluid thermal conductivity and heat transfer enhancements. Heat Transfer Engineering 29(5): 432-460

[26] S J Liao 1995 An approximate solution technique not depending on small parameters: a special example. International Journal of Non-Linear Mechanics 30(3): 371-380

[27] Nayfeh A H 2011 Introduction to perturbation techniques. John Wiley \& Sons

[28] Shijun L 1998 Homotopy analysis method: a new analytic method for nonlinear problems. Applied Mathematics and Mechanics 19(10): 957-962

[29] He J H 2000 Variational iteration method for autonomous ordinary differential systems. Applied Mathematics and Computation 114(2): 115-123

[30] He J H 1999 Homotopy perturbation technique. Computer Methods in Applied Mechanics and Engineering 178(3): 257-262

[31] Elnaqeeb T, Mekheimer K S and Alghamdi F 2016 Cu-blood flow model through a catheterized mild stenotic artery with a thrombosis. Mathematical Biosciences 282: 135-146

[32] Ellahi R, Rahman S U, Mudassar Gulzar M, Nadeem S and Vafai S 2014 A mathematical study of non-Newtonian micropolar fluid in arterial blood flow through composite stenosis. Applied Mathematics and Information Sciences 8(4): 1567-1573

[33] Einstein A 1956 Investigations on the theory of the Brownian movement. Courier Corporation

[34] Brinkman H C 1952 The viscosity of concentrated suspensions and solutions. The Journal of Chemical Physics 20(4): 571-571

[35] Batchelor G K 1977 The effect of Brownian motion on the bulk stress in a suspension of spherical particles. Journal of Fluid Mechanics 83(01): 97-117

[36] Hatami M, Hatami J and Ganji D D 2014 Computer simulation of MHD blood conveying gold nanoparticles as a third grade non-Newtonian nanofluid in a hollow porous vessel. Computer Methods and Programs in Biomedicine 113(2): 632-641

[37] Pak B C and Cho Y I 1998 Hydrodynamic and heat transfer study of dispersed fluids with submicron metallic oxide particles. Experimental Heat Transfer 11(2): 151-170

[38] Godson L, Raja B, Mohan Lal D and Wongwises S 2010 Experimental investigation on the thermal conductivity and viscosity of silver-deionized water nanofluid. Experimental Heat Transfer 23(4): 317-332

[39] Hamilton R L and Crosser O K 1962 Thermal conductivity of heterogeneous two-component systems. Industrial \& Engineering Chemistry Fundamentals 1(3): 187-191

[40] Bennett L 1967 Red cell slip at a wall in vitro. Science 155(3769): 1554-1556

[41] Chaturani P and Biswas D 1984 A comparative study of Poiseuille flow of a polar fluid under various boundary conditions with applications to blood flow. Rheologica Acta 23(4): 435-445 